\title{
Outflows in the narrow-line region of bright Seyfert galaxies - I. GMOS-IFU data
}

\author{
I. C. Freitas, ${ }^{1,2 \star}$ R. A. Riffel, ${ }^{1}$ T. Storchi-Bergmann, ${ }^{3}$ M. Elvis, ${ }^{4}$ A. Robinson, ${ }^{5}$ \\ D. M. Crenshaw ${ }^{6}$ N. M. Nagar, ${ }^{7}$ D. Lena, ${ }^{8,9}$ H. R. Schmitt ${ }^{10}$ and S. B. Kraemer ${ }^{11}$ \\ ${ }^{1}$ Departamento de Física, Universidade Federal de Santa Maria, Centro de Ciências Naturais e Exatas, 97105-900, Santa Maria, RS, Brazil \\ ${ }^{2}$ Universidade Federal de Santa Maria, Colégio Politécnico, 97105-900, Santa Maria, RS, Brazil \\ ${ }^{3}$ Instituto de Física, Universidade Federal do Rio Grande do Sul, CP 15051, 91501-970, Porto Alegre, RS, Brazil \\ ${ }^{4}$ Harvard-Smithsonian Center for Astrophysics, 60 Garden Street, Cambridge, MA 02138, USA \\ ${ }^{5}$ School of Physics and Astronomy, Rochester Institute of Technology, 84 Lomb Memorial Drive, Rochester, NY 14623-5603, USA \\ ${ }^{6}$ Department of Physics and Astronomy, Georgia State University, Astronomy Offices, 25 Park Place, Suite 605, Atlanta, GA 30303, USA \\ ${ }^{7}$ Department of Astronomy, Universidad de Concepción, Casilla 160-C, Concepción, Chile \\ ${ }^{8}$ SRON, Netherlands Institute for Space Research, Sorbonnelaan 2, NL-3584 CA Utrecht, the Netherlands \\ ${ }^{9}$ Department of Astrophysics/IMAPP, Radboud University, Nijmegen, PO Box 9010, NL-6500 GL Nijmegen, the Netherlands \\ ${ }^{10}$ Remote Sensing Division, Naval Research Laboratory, 4555 Overlook Avenue, SW, Washington, DC 20375, USA \\ ${ }^{11}$ Institute for Astrophysics and Computational Sciences, Department of Physics, The Catholic University of America, Washington, DC 20064, USA
}

Accepted 2018 February 1. Received 2018 January 9; in original form 2017 September 12

\begin{abstract}
We present two-dimensional maps of emission-line fluxes and kinematics, as well as of the stellar kinematics of the central few kpc of five bright nearby Seyfert galaxies - Mrk 6, Mrk 79, Mrk 348, Mrk 607, and Mrk 1058 - obtained from observations with the Gemini Multi-Object Spectrograph Integral Field Unit on the Gemini North Telescope. The data cover the inner 3.5 arcsec $\times 5.0$ arcsec - corresponding to physical scales in the range $0.6 \times 0.9-1.5 \times 2.2 \mathrm{kpc}^{2}$ - at a spatial resolution ranging from 110 to $280 \mathrm{pc}$ with a spectral coverage of $4300-7100 \AA$ and velocity resolution of $\approx 90 \mathrm{~km} \mathrm{~s}^{-1}$. The gas excitation is Seyfert like everywhere but show excitation gradients that are correlated with the gas kinematics, reddening and/or the gas density. The gas kinematics show in all cases two components: a rotation one similar to that observed in the stellar velocity field, and an outflow component. In the case of Mrk607, the gas is counter-rotating relative to the stars. Enhanced gas velocity dispersion is observed in association with the outflows according to two patterns: at the locations of the highest outflow velocities along the ionization axis or perpendicularly to it in a strip centred at the nucleus that we attribute to an equatorial outflow. Bipolar outflows are observed in Mrk 348 and Mrk 79, while in Mrk 1058 only the blueshifted part is clearly observed, while in cases of Mrk 6 and Mrk 607, the geometry of the outflow needs further constraints from modelling to be presented in a forthcoming study, where the mass flow rate and powers will also be obtained.
\end{abstract}

Key words: galaxies: active-galaxies: individual: (Mrk 6, Mrk 79, Mrk 348, Mrk 607, Mrk 1058) - galaxies: ISM - galaxies: kinematics and dynamics - galaxies: Seyfert.

\section{INTRODUCTION}

The physical processes that couple the growth of supermassive black holes (SMBHs) to their host galaxies - the so-called feeding and feedback processes - occur in the vicinity of the galaxy nucleus (inner $\approx 1 \mathrm{kpc}$, Hopkins \& Quataert 2010) when it becomes active due to mass accretion to the SMBH (Ferrarese \& Ford 2005; Kormendy \& Ho 2013). The radiation emitted by active galactic nuclei (AGNs) works as a flashlight that illuminates and ionizes

^E-mail: izabelfisica@gmail.com the gas in the vicinity of the nucleus, forming the narrow-line region (NLR). Accretion disc winds (Elvis 2000; Ciotti, Ostriker \& Ostriker 2010) interact with the gas and produce outflows that are observed in the NLR reaching velocities of hundred of $\mathrm{km} \mathrm{s}^{-1}$ (Das et al. 2006; Storchi-Bergmann et al. 2010). Relativistic jets emanating from the AGN also interact with the gas of the NLR. Both types of outflow produce feedback, which is a necessary ingredient in galaxy evolution models to avoid producing overmassive galaxies (Fabian 2012). Inflows have also been observed (e.g. Tacconi et al. 1994; Riffel et al. 2008; Riffel, Storchi-Bergmann \& Winge 2013; Müller-Sánchez et al. 2009; Crenshaw et al. 2010; Schnorr-Müller et al. 2011, 2014a). The importance of the NLR stems from the fact 
that it is spatially resolved and exhibits strong line emission, allowing the observation of the effects of feeding and feedback occurring in this region.

The first imaging studies of the NLR (e.g. Wilson \& Tsvetanov 1994) showed fan-shaped regions, supporting the Unified Model (e.g. Antonucci 1993) and long-slit spectroscopy revealed outflows along the cones (Storchi-Bergmann, Wilson \& Baldwin 1992; Das et al. 2006; Crenshaw et al. 2010). However, Hubble Space Telescope (HST) [O III] images of the NLR of a complete sample based on $60 \mu \mathrm{m}$ luminosity revealed that the conical morphology is more the exception than the rule (Schmitt et al. 2003). A recent long-slit HST Space Telescope Imaging Spectrograph (STIS) study was conducted on a sample of 48 AGN, 35 of which showed extended NLR (Fischer et al. 2013). Only 1/3 (12) of that sub-sample have outflowing kinematics, while the remaining are classified as 'ambiguous' or 'complex'.

Integral Field Spectroscopy (IFS) using large telescopes is a powerful tool to map the NLR of nearby galaxies (e.g. Riffel et al. 2006; Barbosa at al. 2009; Riffel \& Storchi-Bergmann 2011a,b; Harrison et al. 2014; Davies et al. 2014; Diniz et al. 2015; Lin et al. 2016; Fischer et al. 2017; Müller-Sánchez et al. 2011, 2017; Wylezalek et al. 2017; da Silva, Steiner \& Menezes 2017; Bae et al. 2017), as they provide the spatial coverage missed by long-slit spectroscopy at resolutions of a few tens of parsecs. We have recently used the Gemini Multi-Object Spectrograph Integral Field Unit (GMOS-IFU), to study the gas distribution, excitation, and kinematics of the inner kiloparsec of a few nearby galaxies, including the Seyfert 2 galaxies NGC 2110 (Schnorr-Müller et al. 2014b) and NGC 1386 (Lena et al. 2014, 2015) as well as Seyfert 1.8 galaxy NGC 1365 (Lena et al. 2016). In these cases, we found that most of the extended NLR emission has kinematics that can be attributed to gas rotating in the galaxy disc. In addition, outflows are observed within the inner $\approx 300$ pc for NGC 2110 , being quasi-spherical rather than conical. NGC 1386 has features that suggest the presence of a bipolar outflow, located within the inner $\approx 150 \mathrm{pc}$. Lena et al. (2016) found that there is a fan-shaped outflow in the NGC 1365, as suggested previously (e.g. Edmunds, Taylor \& Turtle 1988). Similar results are found by studies in the near-infrared (near-IR), which suggest that the molecular and ionized gas kinematics of the inner few hundred of parsecs of nearby Seyfert galaxies present a combination of both rotation and in gas outflows (e.g. Fischer et al. 2017; Riffel et al. 2013). IFS is the best way to constrain the structure and kinematics of the NLR. A complete census of the feeding and feedback processes is still lacking.

In this paper, we use IFS to study the gas distribution and kinematics in the inner kpc of five nearby AGNs: Mrk 6, Mrk 79, Mrk 348, Mrk 607, and Mrk 1058. Previous optical IFS is available only for Mrk 348 of our sample (Stoklasová et al. 2009), but with a lower spatial resolution than that presented here. We present and discuss flux and emission-line ratio maps, as well as maps of the gas velocity fields and velocity dispersion. A detailed analysis of the gas kinematics will be presented in a forthcoming paper. In Section 2, we describe the observations and data reduction procedures. In Section 3, we present the emission-line flux and kinematics maps, which are discussed in Section 4. The conclusions of this work are presented in Section 5.

\section{OBSERVATIONS AND DATA REDUCTION}

We have selected our sample from the Seyfert galaxies observed in the HST [O III] Snapshot Survey (Schmitt et al. 2003), that comprises $60 / 88$ of all Seyfert galaxies with $z \leq 0.031$ from the catalogue of warm IRAS sources (de Grijp et al. 1992). From the 60 galaxies, we selected a sub-sample of 30 according to the following criteria: (1) each galaxy presents extended emission beyond $\approx 1.0$ arcsec from the nucleus; (2) the sub-sample spanned the whole range of AGN luminosities from the sample $\left(39 \leq \log \left(\mathrm{L}[\mathrm{O}\right.\right.$ III] $\left.) \leq 42 \mathrm{erg} \mathrm{s}^{-1}\right)$. We excluded already well-studied objects, such as NGC 4151 and NGC 1068, and also targets already observed by members of our groups in previous runs. In this work, we present a study using new data for the galaxies Mrk 6, Mrk 79, Mrk 348, Mrk 607, and Mrk 1058.

We used the GMOS operating in the IFU mode on the Gemini North Telescope. The observations were made from 2014 September 30 to 2015 January 12 under the project GN-2014BQ-87. We used the IFU on the one-slit mode and two sets of observations were made, one centred at $5700 \AA$ and another at $5750 \AA$, to correct for the effects of the gaps between the GMOS CCDs. The B600 grating was used in combination with the G5307 filter in order to obtain spectra in the range from 4300 to $7100 \AA$, that includes the most intense emission lines from the NLR of AGNs: $\mathrm{H} \beta$, [O III] $\lambda \lambda 4959,5007$, [O I] $\lambda 6300, \mathrm{H} \alpha$, $[\mathrm{N}$ II] $\lambda \lambda 6548,83$ and $[\mathrm{S}$ II] $\lambda \lambda 6716,31$. The number of individual exposures for each galaxy can be found in Table 1 and the total exposure time ranged from 81 to $95 \mathrm{~min}$.

Data reduction was performed using a series of tasks from the GMOS package developed as part of the GEMINI IRAF package. The reduction process followed the standard procedure of GMOS-IFU data reduction (see Lena 2014) including bias and sky subtraction, flat-fielding, trimming of the images, wavelength and flux calibration, building of the data cubes, final alignment, and combination of individual exposure cubes. The individual data cubes were median combined using the position of the peak of the continuum emission as reference to generate the final data cube for each galaxy.

In Table 1, we present the distance as quoted in NED, ${ }^{1}$ nuclear activity class, the spatial and spectral resolutions, the exposure time, 2-10 keV X-ray luminosity, [O III] luminosity by Schmitt et al. (2003), total [O III] luminosity obtained from the our data and IFU position angle (PA). The spectral resolution was estimated by measuring the full width at half-maximum (FWHM) of emission-line profiles of the $\mathrm{CuAr}$ calibration lamp, used to wavelength calibrate the data. The angular resolution was estimated as the FWHM of the continuum flux distribution of field stars from the acquisition images.

\section{MEASUREMENTS}

\subsection{Emission-line profile fitting}

Fig. 1 presents the nuclear spectra for the five galaxies of our sample, integrated within square apertures of $0.25 \operatorname{arcsec} \times 0.25$ arcsec, centred at the peak of the continuum emission. The strongest emission lines are identified in each spectrum that shows numerous emission lines (up to about 20).

In order to map the emission-line flux distributions, line-of-sight velocity $\left(V_{\mathrm{LOS}}\right)$ and velocity dispersion $(\sigma)$, we fitted the line profiles at each spaxel by Gaussian curves using adapted versions of the emission-line PROfile FITting routine (profit Riffel 2010), which uses the MPFITFUN routine (Markwardt et al. 2009), to perform the non-linear least-squares fit.

\footnotetext{
${ }^{1}$ NASA/IPAC Extragalactic Database, available at http://ned.ipac. caltech.edu.
} 
Table 1. Observations log.

\begin{tabular}{|c|c|c|c|c|c|c|c|c|c|}
\hline Object & $\begin{array}{c}\text { Distance } \\
(\mathrm{Mpc})\end{array}$ & $\begin{array}{l}\text { Nuclear } \\
\text { activity }\end{array}$ & $\begin{array}{l}\text { Spatial } \\
\text { resolution } \\
\quad(\mathrm{pc})\end{array}$ & $\begin{array}{l}\text { Spectral } \\
\text { resolution } \\
\left(\mathrm{km} \mathrm{s}^{-1}\right)\end{array}$ & $\begin{array}{l}\text { Exposure } \\
\text { time } \\
(\mathrm{s})\end{array}$ & $\begin{array}{c}\log L_{2-10 \mathrm{keV}} \\
\left(\mathrm{erg} \mathrm{s}^{-1}\right)\end{array}$ & $\begin{array}{c}\log L_{[\mathrm{O} \mathrm{III]}}{ }^{a} \\
\quad\left(\operatorname{erg~s}^{-1}\right)\end{array}$ & $\begin{array}{c}\log L_{[\mathrm{OIII}]} \\
\left(\operatorname{erg~s}^{-1}\right)\end{array}$ & $\begin{array}{c}\text { IFU position } \\
\text { angle } \\
\left({ }^{\circ}\right)\end{array}$ \\
\hline Mrk 6 & 79.0 & Sy 1.5 & 235 & 90 & $7 \times 810$ & $43.0^{b}$ & 41.72 & 42.24 & 280 \\
\hline Mrk 79 & 91.6 & Sy 1 & 280 & 90 & $6 \times 810$ & $43.4^{c}$ & 41.58 & 41.95 & 73 \\
\hline Mrk 348 & 63.9 & Sy 2 & 190 & 85 & $6 \times 810$ & $42.6^{d}$ & 41.26 & 41.91 & 185 \\
\hline Mrk 607 & 36.1 & Sy 2 & 110 & 90 & $7 \times 810$ & $40.8^{e}$ & 40.44 & 41.02 & 137 \\
\hline Mrk 1058 & 71.8 & Sy 2 & 215 & 85 & $6 \times 810$ & - & 40.49 & 41.23 & 121 \\
\hline
\end{tabular}

References: ${ }^{a}$ Schmitt et al. (2003); ${ }^{b}$ Lutz et al. (2004); ${ }^{c}$ Kaspi et al. (2005); ${ }^{d}$ Ueda et al. (2001); ${ }^{e}$ LaMassa et al. (2011).

The following fitting procedure was adopted: the $[\mathrm{N}$ II] $\lambda \lambda 6548$, $83+\mathrm{H} \alpha$ emission lines were fitted by keeping the $[\mathrm{N}$ II] flux ratio fixed ([N $\mathrm{NI}] \lambda 6583 /[\mathrm{N}$ II] $\lambda 6548=3$ ) and tying the central wavelength and width of the $\left[\mathrm{N}_{\mathrm{II}}\right]$ lines for all galaxies. For Mrk 6 and Mrk 79, the same procedure was adopted to fit the [O III] $\lambda \lambda 4959,5007+\mathrm{H} \beta$ profiles, setting the flux ratio [O III] $\lambda 5007 /[\mathrm{O}$ III] $\lambda 4959=3$ and tying kinematics of the [O III $]$ lines, while for Mrk 348 and Mrk 1058, the $\mathrm{H} \beta$ profile was fitted individually and the $[\mathrm{O}$ III] $\lambda \lambda 4959,5007$ profiles were fitted simultaneously by using the same constraints above. Finally, for Mrk 607, the $[\mathrm{O}$ III] and $\mathrm{H} \beta$ lines were fitted individually. These distinct procedures to fit the [O III] $\lambda \lambda 4959,5007+\mathrm{H} \beta$ profiles among the galaxies of our sample were adopted in order to have the minimum number of free parameters as possible, resulting in better constrained measurements. The choice of fitting the emission lines individually or simultaneously was done by a visual inspection of the spectra and resulting fits for each galaxy. In all cases, where the emission-line profiles are separated with adjacent continuum regions large enough to constrain its slope, the fit of individual components resulted in better models for the observed profiles. The [S II] doublet was fitted by keeping the kinematics of the two lines tied, while other emission lines were fitted individually with all parameters free.

As the spectral range used to fit each line profile is small, we fitted the underlying continuum by a linear equation. Although the line profiles at most locations for all galaxies are well reproduced by single Gaussian components, double or multiple kinematic components are seen at some locations, in particular for Mrk 348 and Mrk 6. A detailed gas kinematics based on multicomponents fits, as well velocity channel maps, will be presented in a forthcoming paper. All maps presented in this paper are based on the fitting of one Gaussian for each emission-line profile. In addition, for the Seyfert 1 galaxies Mrk 6 and Mrk 79, we included broad components to fit the $\mathrm{H} \alpha$ and $\mathrm{H} \beta$ profiles to account for the broad-line region (BLR) emission. Besides the measurement for each parameter, the fitting routine outputs their uncertainties (as estimated by the MPFITFUN routine) and the resulting $\chi^{2}$ of the best fit.

In Fig. 2, we present examples of the fits of the emission-line profiles for the nuclear spectrum of each galaxy, identified at the top-left corner of each panel. In case of the Seyfert 1 galaxies, as the broad profiles were clearly non-Gaussian, we had to use two or more components to represent these profiles, and these components, together with the narrow lines are shown as doted blue lines in the Fig. 2.

Although several emission lines are observed for all objects, we constructed maps for the flux distributions and kinematics of the following emission lines: $\left[\mathrm{O}_{\mathrm{III}}\right] \lambda 5007,\left[\mathrm{O}_{\mathrm{I}}\right] \lambda 6300, \mathrm{H} \alpha,\left[\mathrm{N}_{\mathrm{II}}\right] \lambda 6583$, and $\left[\mathrm{S}_{\mathrm{II}}\right] \lambda 6731$. These particular lines were chosen because they present the highest signal-to-noise $(\mathrm{S} / \mathrm{N})$ ratio among their species.
In addition, we fitted also the $\mathrm{H} \beta$ profile, as it can be used together with the $\mathrm{H} \alpha$ flux to estimate the reddening.

\subsection{Stellar kinematics}

We used the penalized pixel-fitting PPXF routine (Cappellari \& Emsellem 2004) to fit the absorption spectra of the galaxies of our sample and obtain measurements for the two-dimensional stellar line-of-sight velocity distribution (LOSVD). Each galaxy spectrum is fitted by convolving spectral templates with the corresponding LOSVD, represented by Gauss-Hermite series. As spectral templates, we used single stellar populations synthetic spectra selected from Bruzual \& Charlot (2003), which have similar spectral resolution to that of the GMOS data.

As the stellar absorption features seen in our sample spectra are usually weak, we have fitted the whole spectral range at each spaxel for all galaxies, by masking regions with strong emission lines. During the fit, we used a truncated second-order Gauss-Hermite series to parametrize the LOSVD, thus resulting in measurements for the stellar LOS velocity $\left(V_{*}\right)$ and velocity dispersion $\left(\sigma_{*}\right)$. In addition, we allowed PPXF to include seventh-order additive Legendre polynomials to better fit the continuum shape and we used the clean parameter to reject all spectral pixels deviating more than $3 \sigma$ from the best fit, in order to exclude spurious features and possible weak emission lines.

As output, the PPXF code returns measurements of the $V_{*}$ and $\sigma_{*}$ at each spatial position and their corresponding uncertainties. These measurements were used to construct two-dimensional maps, which are presented in the next section together with the emission-line maps. For Mrk 6, the S/N ratio of the absorption spectra was not high enough to obtain reliable measurements of the stellar kinematics and thus, we do not present maps for this galaxy.

\section{RESULTS}

In Figs 3-7, we present two-dimensional maps for the flux distributions, gas and stellar kinematics, and line ratios for the galaxies of our sample. Each figure is organized as follows:

(i) Top left panel: large-scale image, obtained with the HST Wide Field Planetary Camera 2 (WFPC2) through the filter F606W (Malkan, Gorjian \& Tam 1998). These images are used to illustrate the large-scale continuum emission of each galaxy. The spatial orientation of our GMOS data is shown in the top-left corner. The colour bar shows the flux in arbitrary units;

(ii) Top second panel (from left to right): [O III] $\lambda 5007 \AA$ narrowband image, obtained with the WFPC2 Linear Ramp Filter (from Schmitt et al. 2003). The colour bar shows the flux in units of $10^{-16} \mathrm{erg} \mathrm{s}^{-1} \mathrm{~cm}^{-2}$. The images cover the inner $6 \operatorname{arcsec} \times$ 

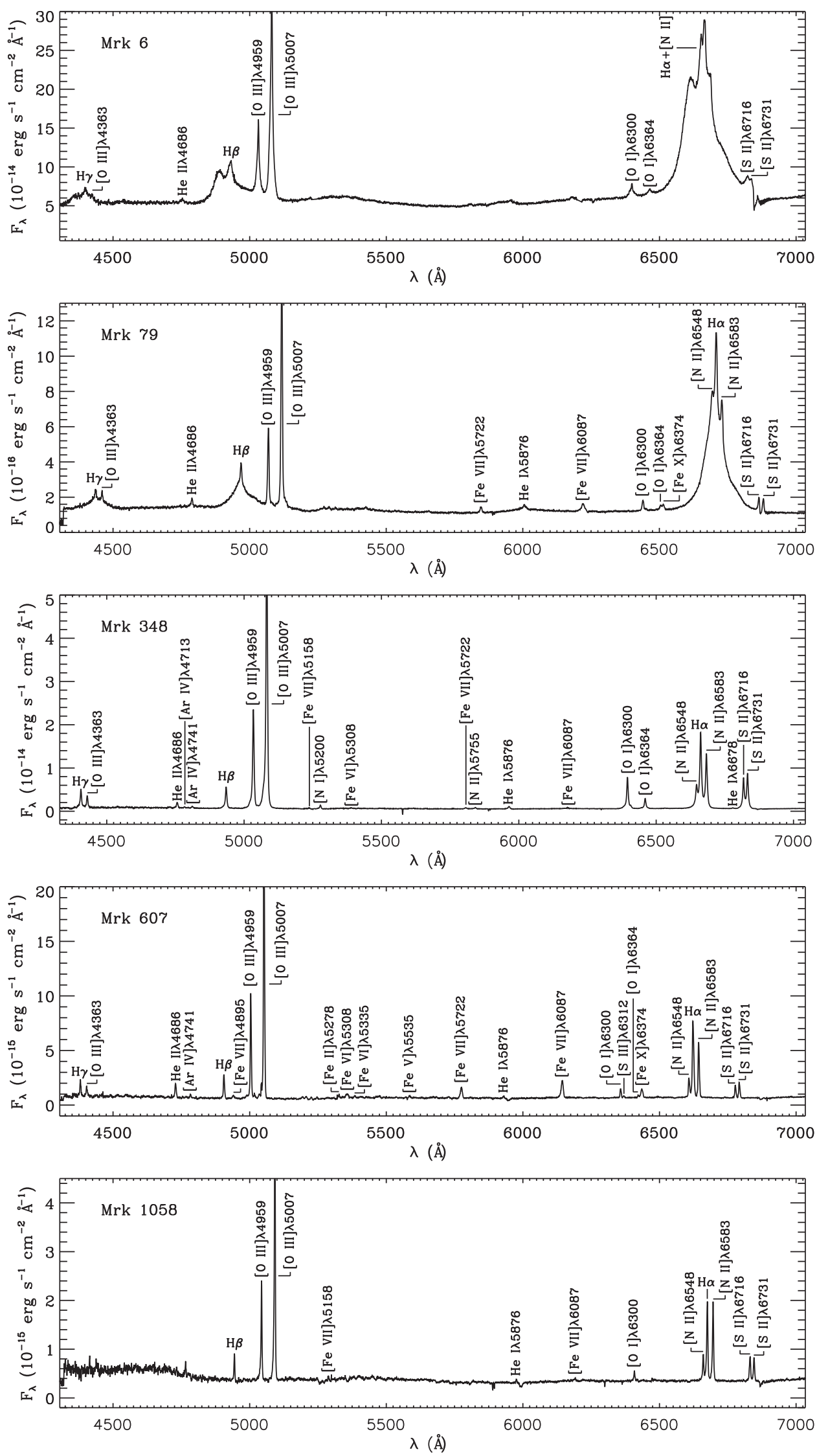

Figure 1. Nuclear spectra of the galaxies of our sample for an aperture of $0.25 \operatorname{arcsec} \times 0.25 \operatorname{arcsec}$. The strongest emission lines are identified at each panel. From top to bottom: Mrk 6, Mrk 79, Mrk 348, Mrk 607, and Mrk 1058. 

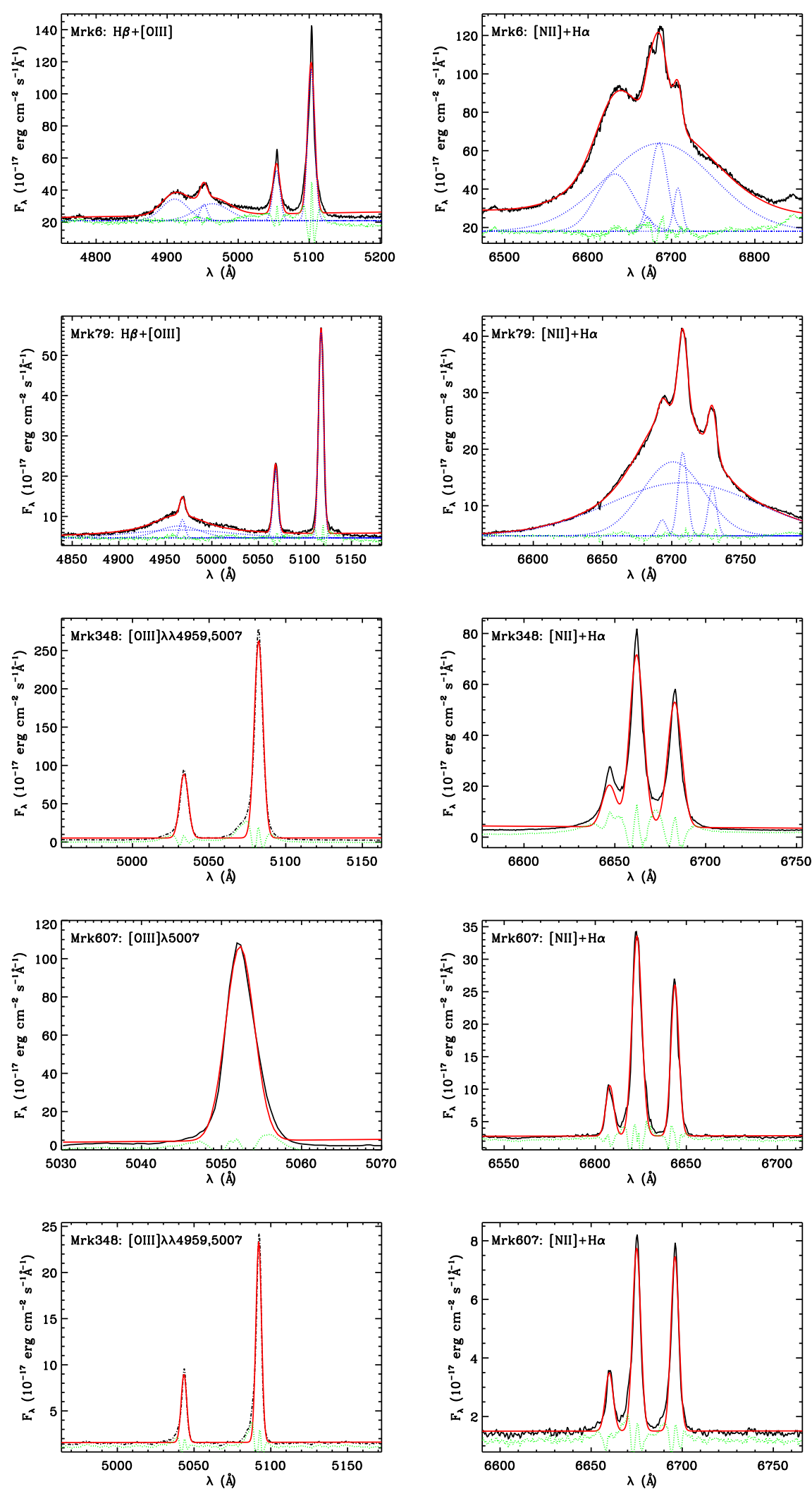

Figure 2. Examples of fits of the emission-line profiles at the nucleus of each galaxy. The galaxy name and fitted emission lines are indicated in the top left corner of each panel. The observed profiles are shown in black, the best model in red, and the dotted green lines show the residuals (observed - model), plus an arbitrary constant. For the Seyfert 1 galaxies (Mrk 6 and Mrk 79), each individual component is shown as dotted blue lines. Flux units $\left(\mathrm{F}_{\lambda}\right)$ are $10^{-17}$ $\operatorname{erg~s}^{-1} \mathrm{~cm}^{-2} \AA^{-1}$. 

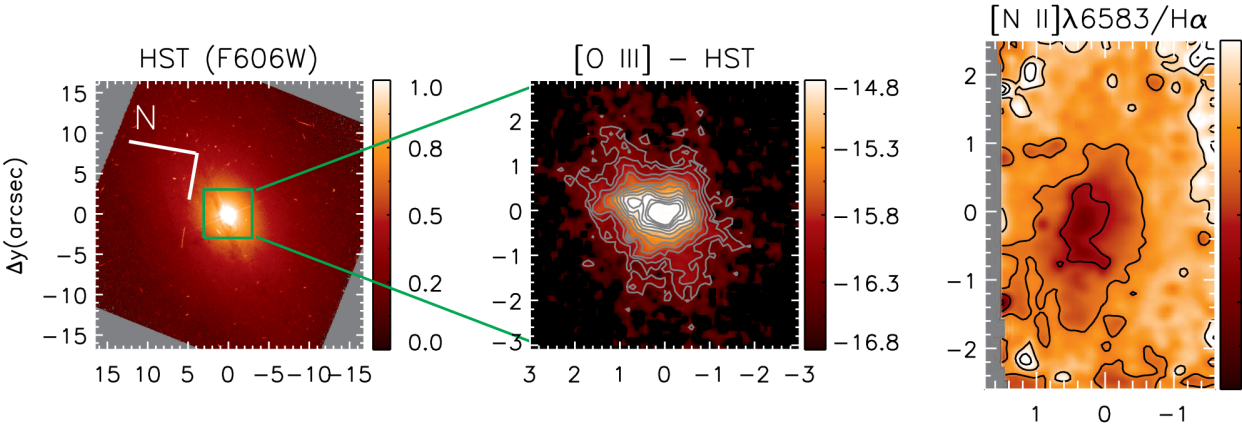

0.9

0.7

0.4

0.2

0.0
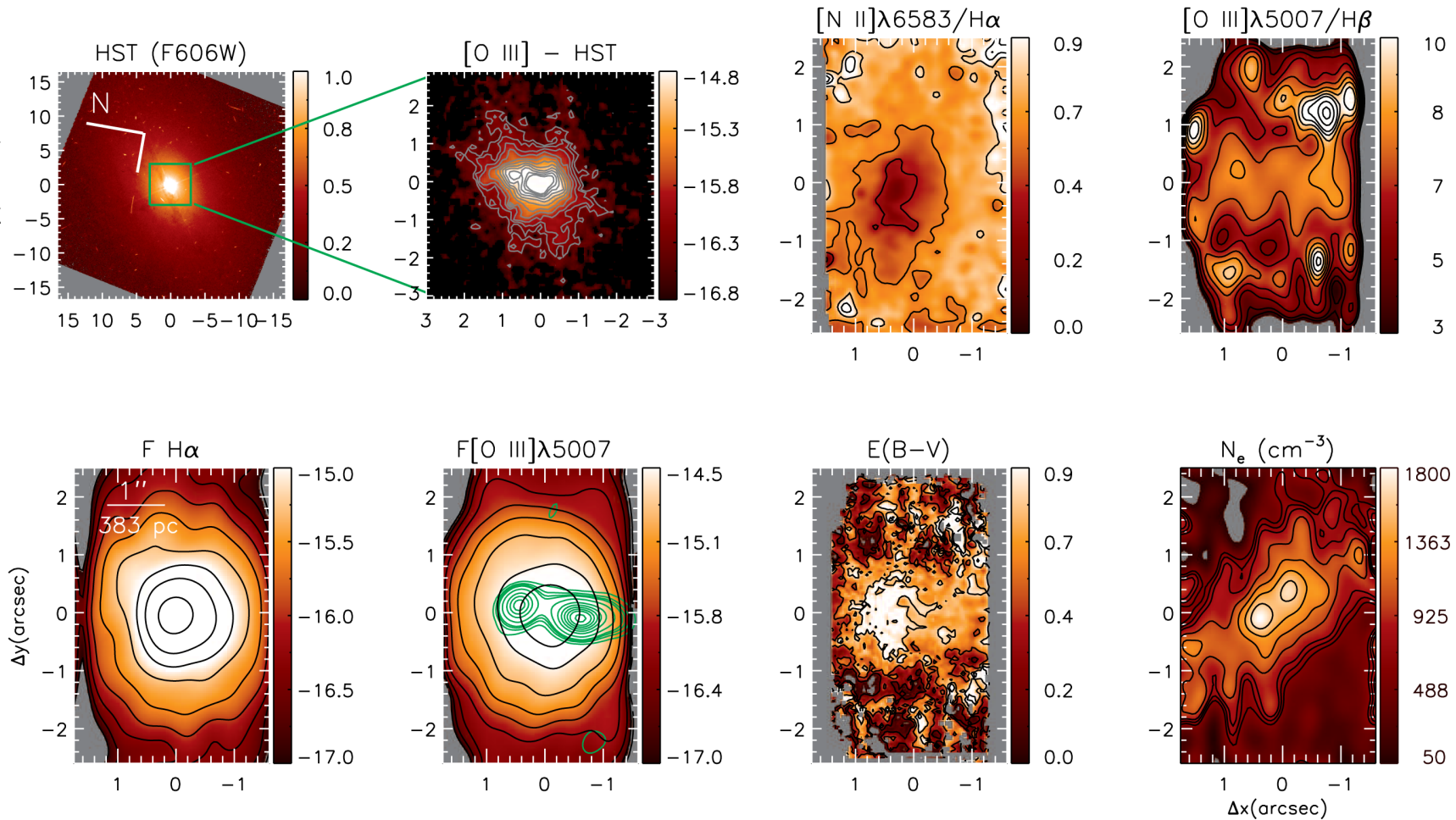
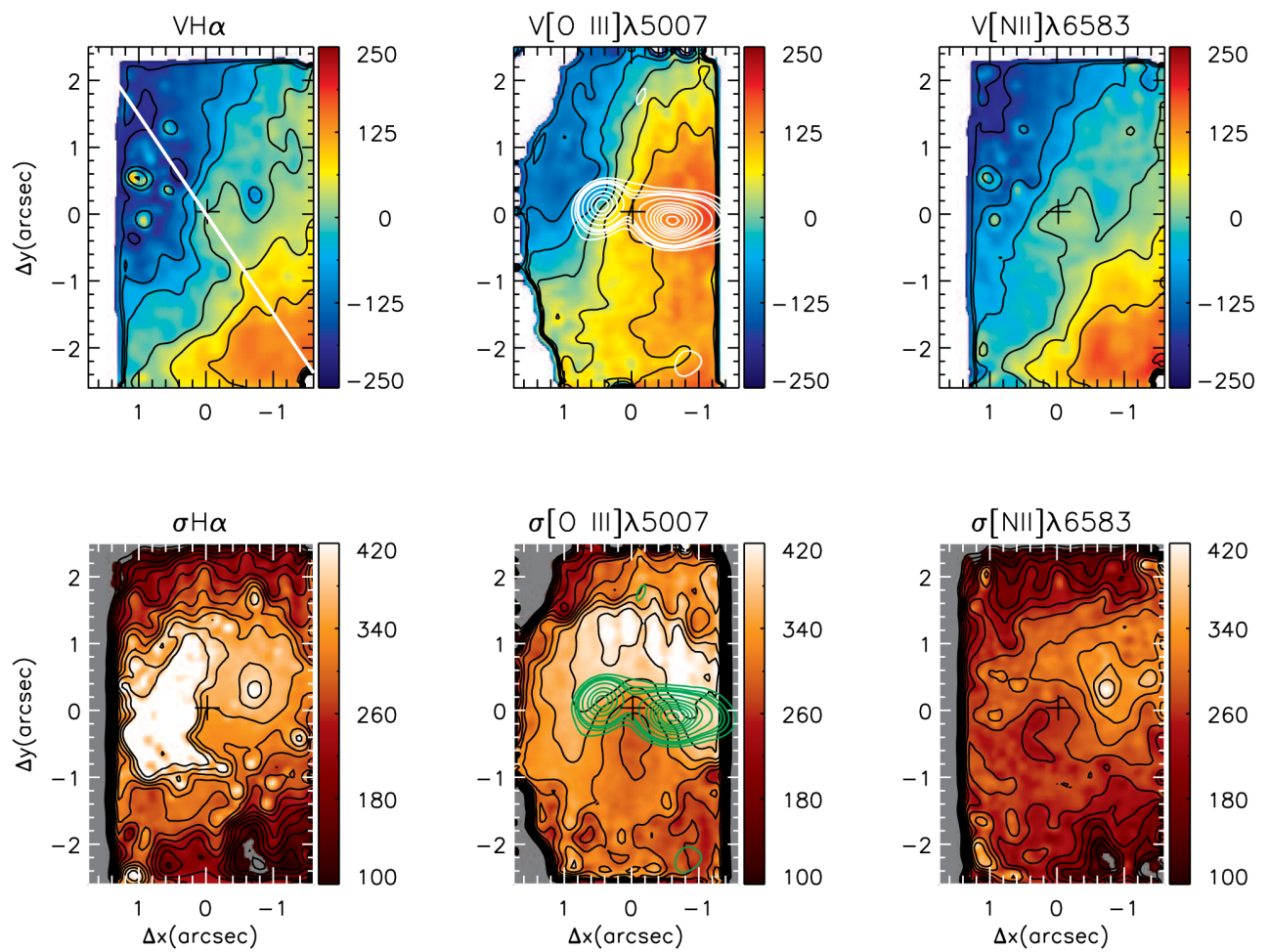

Figure 3. Two-dimensional maps for Mrk 6 organized as described in Section 4 . The central cross marks the location of the nucleus, defined as the position of the peak of continuum emission and the spatial orientation is shown at the top left corner of the large-scale image. The contours overplotted to the [O III] $\lambda 5007$ flux map (in green), velocity field (in white), and $\sigma$ map (green) are from the $3.6 \mathrm{~cm}$ radio image of Schmitt et al. (2001). The white line shown in the $\mathrm{H} \alpha$ velocity field represents the major axis of the large-scale disc, measured using I-band images by Schmitt \& Kinney (2000). Grey regions in the flux, ratio, and velocity dispersion maps and white regions in the velocity fields correspond to locations where the $\mathrm{S} / \mathrm{N}$ ratio was not high enough to obtain a good fit of the line profiles. For this galaxy, we were not able to obtain reliable measurements of the stellar kinematics. 

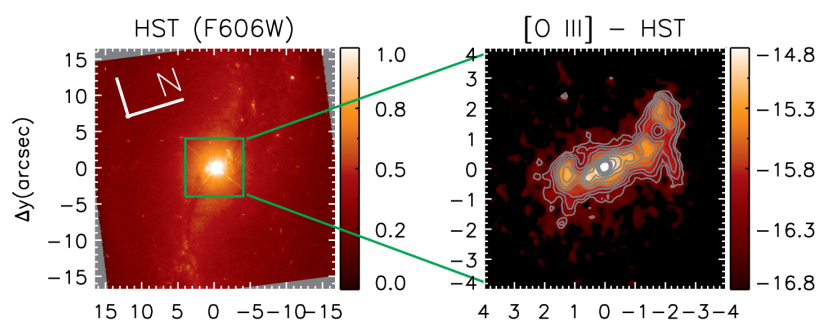

$\mathrm{FH} \alpha$
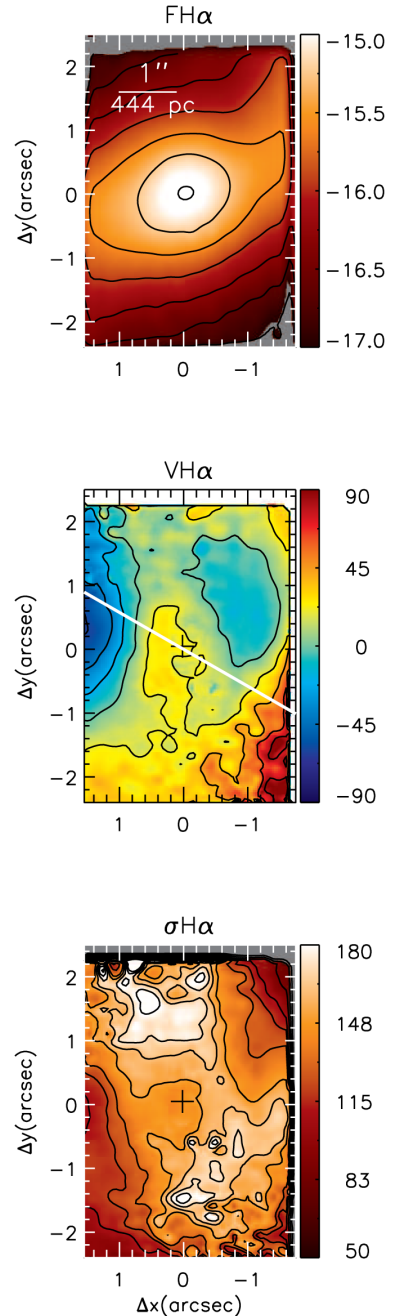

$\mathrm{F}[\mathrm{O} \quad \mathrm{III}] \lambda 5007$
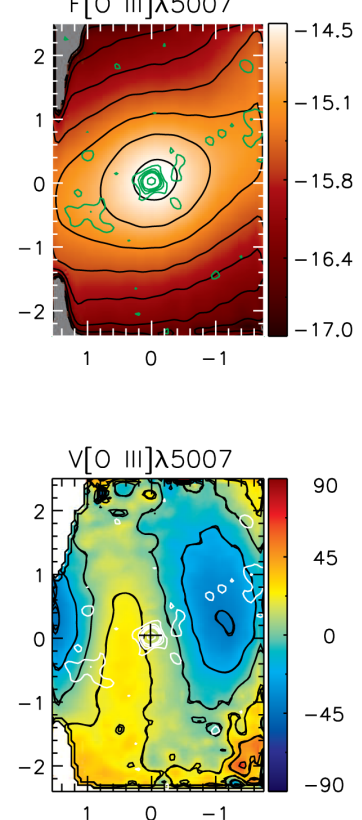

$[\mathrm{N} \mathrm{II}] \lambda 6583 / \mathrm{H \alpha}$
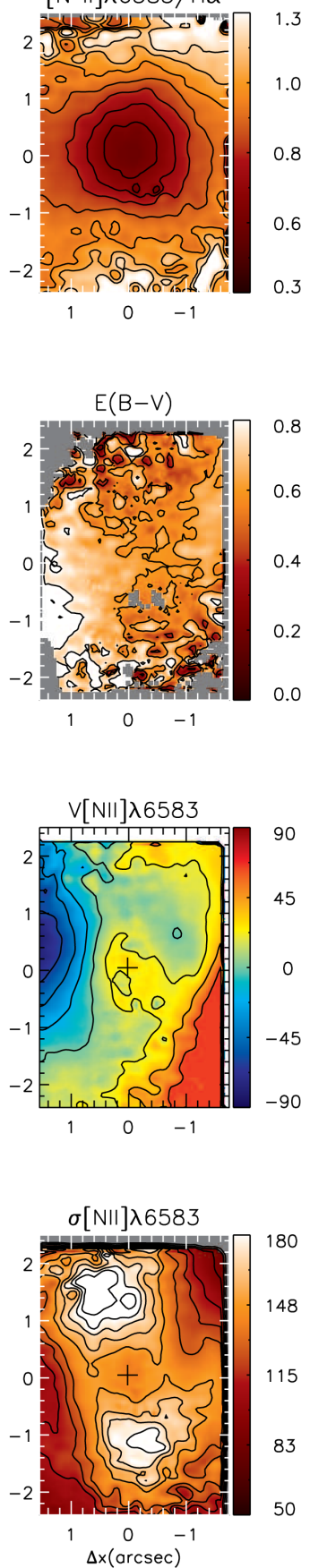
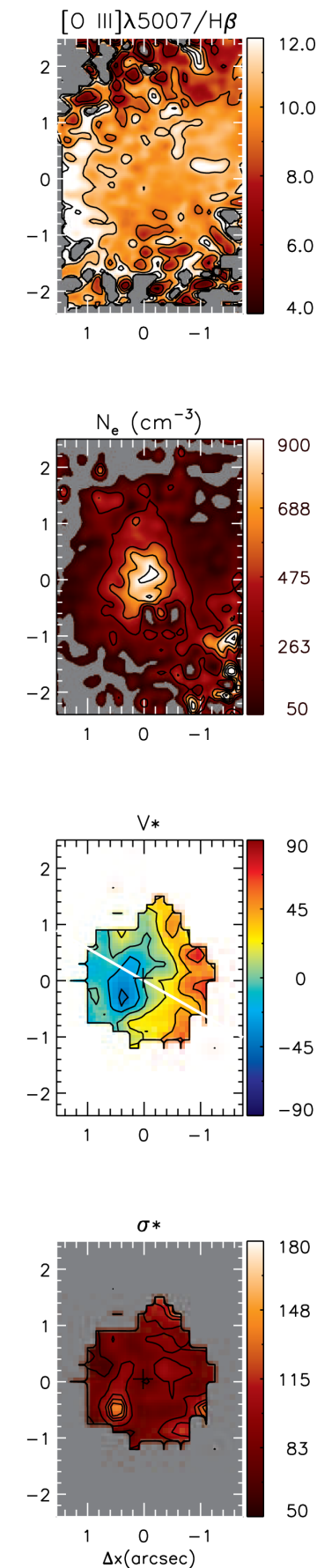

Figure 4. Same as Fig. 3 for Mrk 79. For this galaxy, we could obtain reliable measurements for the stellar kinematics within the inner 1 arcsec, and the corresponding stellar velocity field $\left(V_{*}\right)$ and velocity dispersion $\left(\sigma_{*}\right)$ are shown in the rightmost bottom panels.

6 arcsec for the galaxies Mrk 6, Mrk 348, and Mrk 607, the inner 8 arcsec $\times 8$ arcsec for Mrk 79 and $4 \operatorname{arcsec} \times 4 \operatorname{arcsec}$ for Mrk 1058; the angular resolution is 0.1 arcsec, about 5 times better than that of our GMOS data;

(iii) Top third panel: $[\mathrm{N}$ II] $\lambda 6583 / \mathrm{H} \alpha$ line ratio map;

(iv) Top right panel: $[\mathrm{O}$ III $] \lambda 5007 / \mathrm{H} \beta$ line ratio map;

(v) Second row, left-hand panel: flux distribution for the $\mathrm{H} \alpha$ emission line, obtained from the fit of the line profile with a Gaussian curve. The colour bar shows the flux in logarithmic units of $\mathrm{erg} \mathrm{s}^{-1} \mathrm{~cm}^{-2}$; (vi) Second row, second panel: flux distribution for the [O III] $\lambda 5007$ emission line, shown in logarithmic units of $\mathrm{erg} \mathrm{s}^{-1} \mathrm{~cm}^{-2}$;

(vii) Second row, third panel: reddening map obtained from the $\mathrm{H} \alpha / \mathrm{H} \beta$ line ratio using the following expression:

$E(B-V)=1.38 \log \left(\frac{\frac{\mathrm{FH}_{\alpha}}{\mathrm{F}_{\mathrm{H}_{\beta}}}}{2.86}\right)$

where $\mathrm{F}_{\mathrm{H} \alpha}$ and $\mathrm{F}_{\mathrm{H} \beta}$ are the observed fluxes of the $\mathrm{H} \alpha$ and $\mathrm{H} \beta$ emission lines, respectively. This expression was obtained considering 

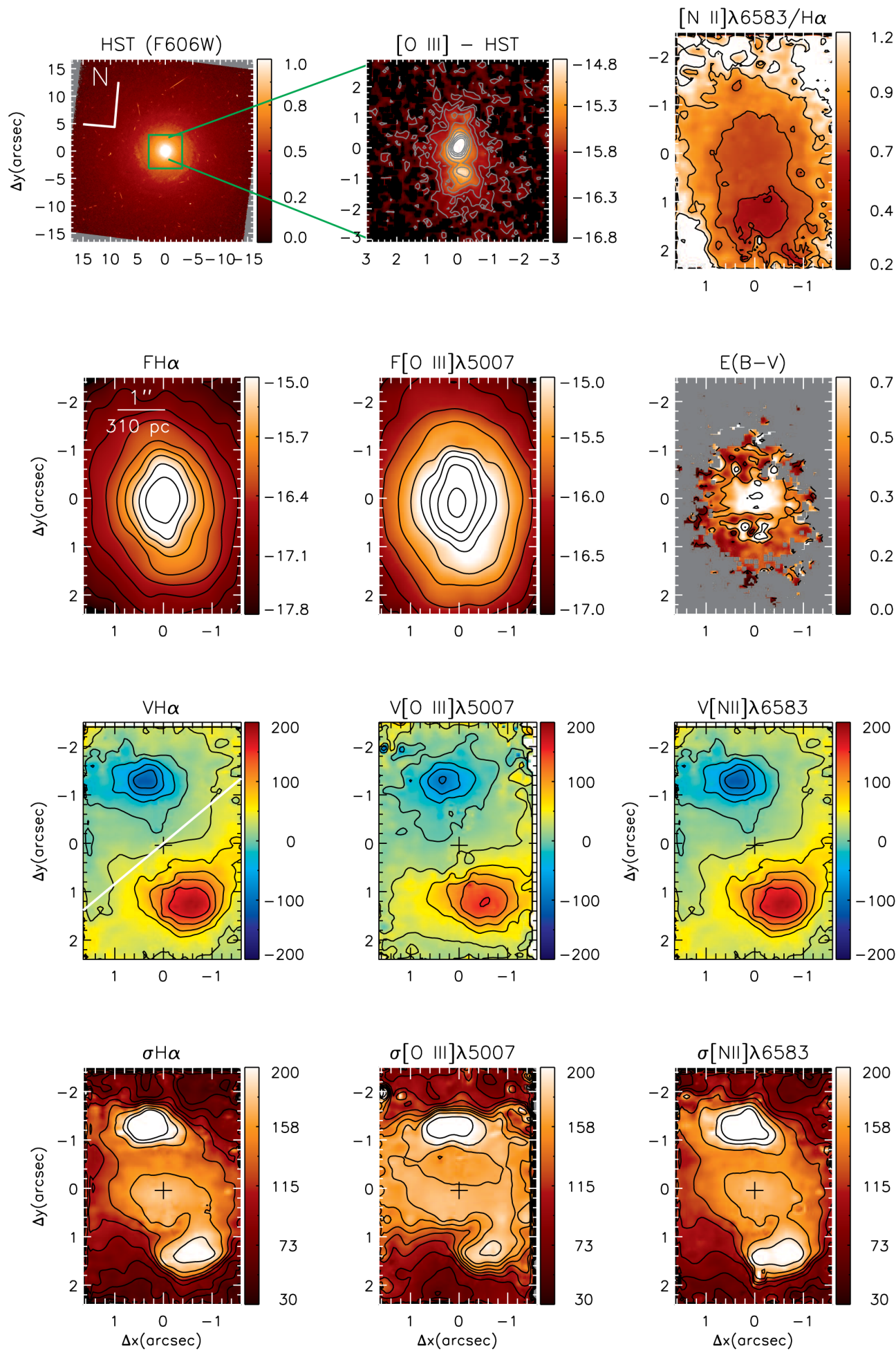
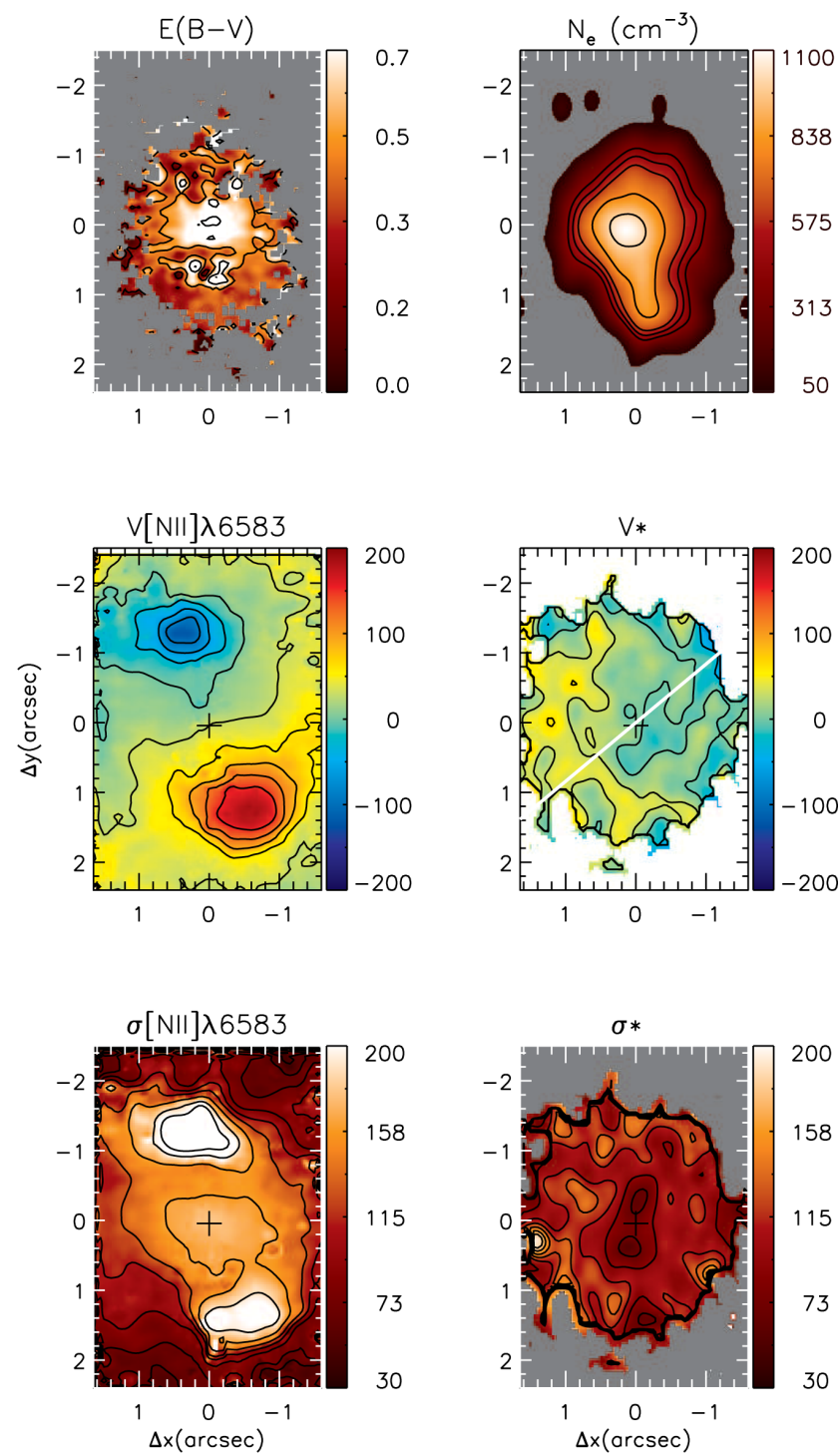

Figure 5. Same as Fig. 4 for Mrk 348.

the reddening law of Cardelli, Clayton \& Mathis (1989) and adopting the theoretical line ratio $\frac{\mathrm{F}_{\mathrm{H} \alpha}}{\mathrm{F}_{\mathrm{H} \beta}}=2.86$, corresponding to the case B of H r recombination for an electron density of $N_{\mathrm{e}}=100 \mathrm{~cm}^{-3}$ and electron temperature of $T_{\mathrm{e}}=10000 \mathrm{~K}$ (Osterbrock \& Ferland 2006);

(viii) Second row, right-hand panel: electron density map obtained from the $[\mathrm{S}$ II] $\lambda \lambda 6716 / 6731$ flux ratio assuming an electron temperature $T_{\mathrm{e}}=10000 \mathrm{~K}$ using the temden IRAF routine;

(ix) Third row, left-hand panel: velocity field for the $\mathrm{H} \alpha$ emitting gas (narrow component). The colour bar shows the $V_{\text {LOS }}$ in units of $\mathrm{km} \mathrm{s}^{-1}$, after the subtraction of the systemic velocity of the galaxy, defined as the velocity measured for the stars from the inner $0.45 \operatorname{arcsec} \times 0.45$ arcsec, with exception of Mrk 6, for which 

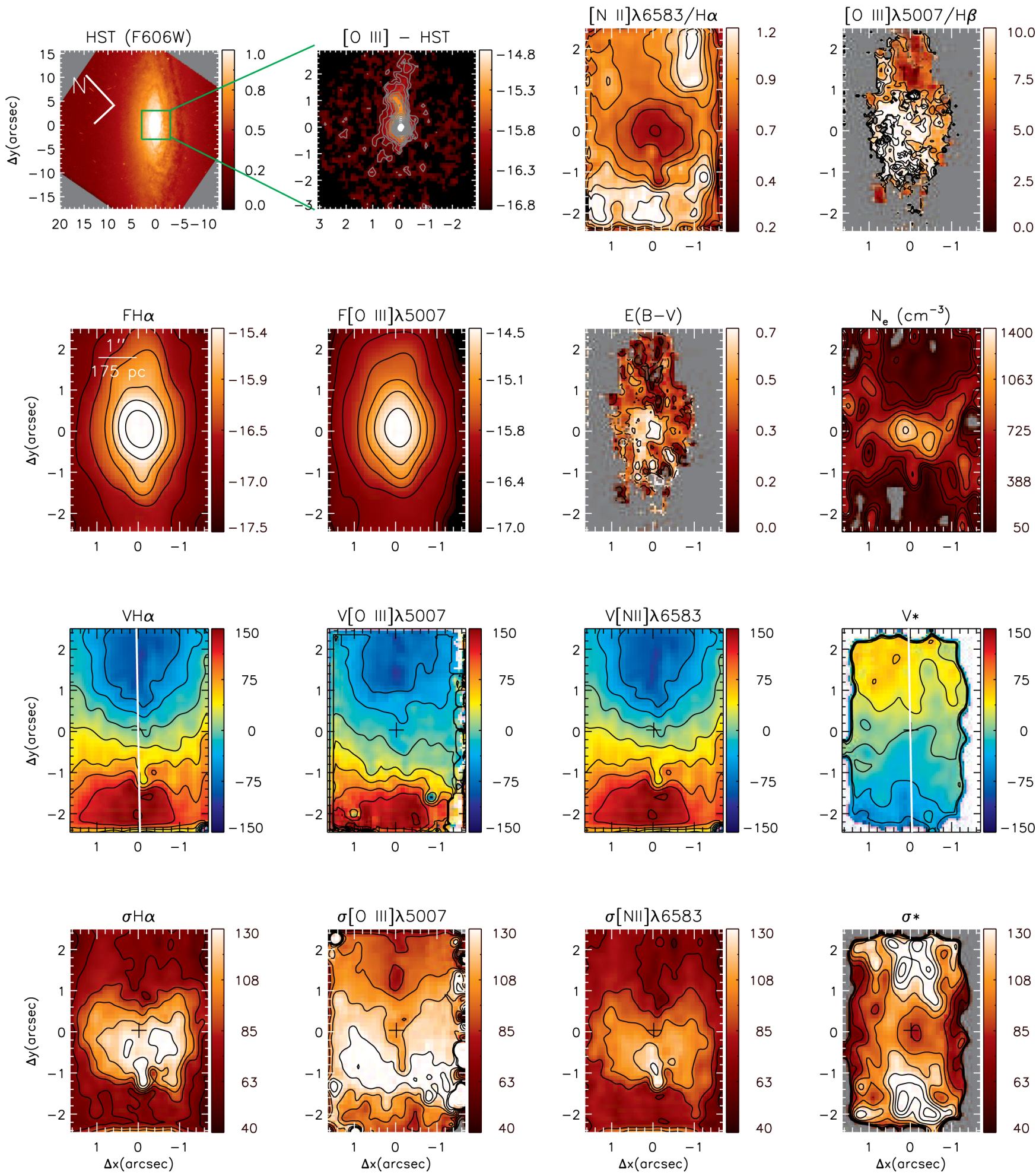

Figure 6. Same as Fig. 4 for Mrk 607.

the systemic velocity is defined as the velocity of the $\mathrm{H} \beta$ narrow component for the same aperture;

(x) Third row, second panel: same as previous panel, for the [O III] $\lambda 5007$ emission line;

(xi) Third row, third panel: same as previous panel, for the [ $\left.\mathrm{N}_{\mathrm{II}}\right]$ $\lambda 6583$ emission line; (xii) Third row, right-hand panel: same as previous panel, for the stars;

(xiii) Bottom left panel: velocity dispersion map for the $\mathrm{H} \alpha$ emission line, with the colour bar showing the $\sigma$ values in units of $\mathrm{km} \mathrm{s}^{-1}$;

(xiv) Bottom second panel: same as previous panel, for the [O III] $\lambda 5007$ emission line; 

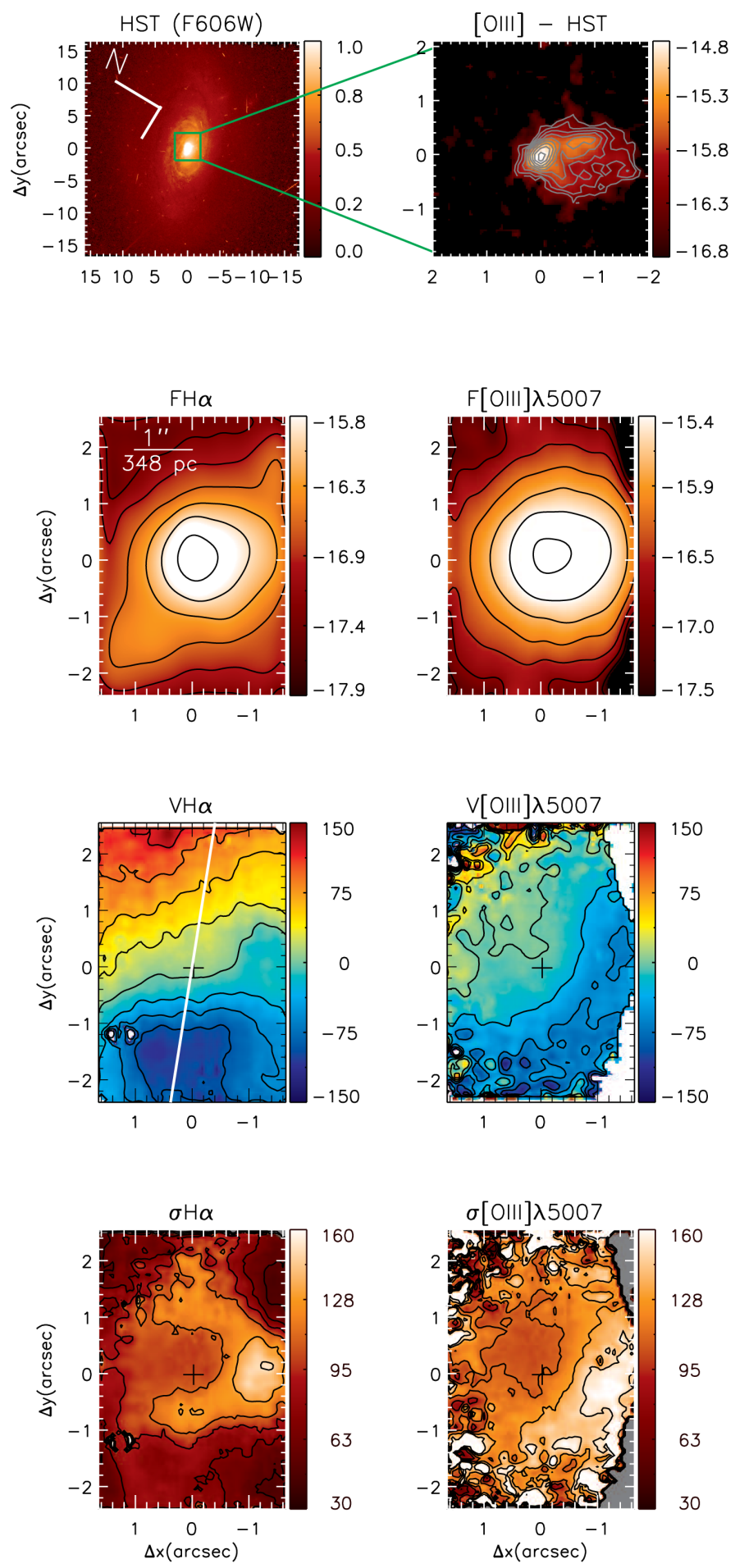
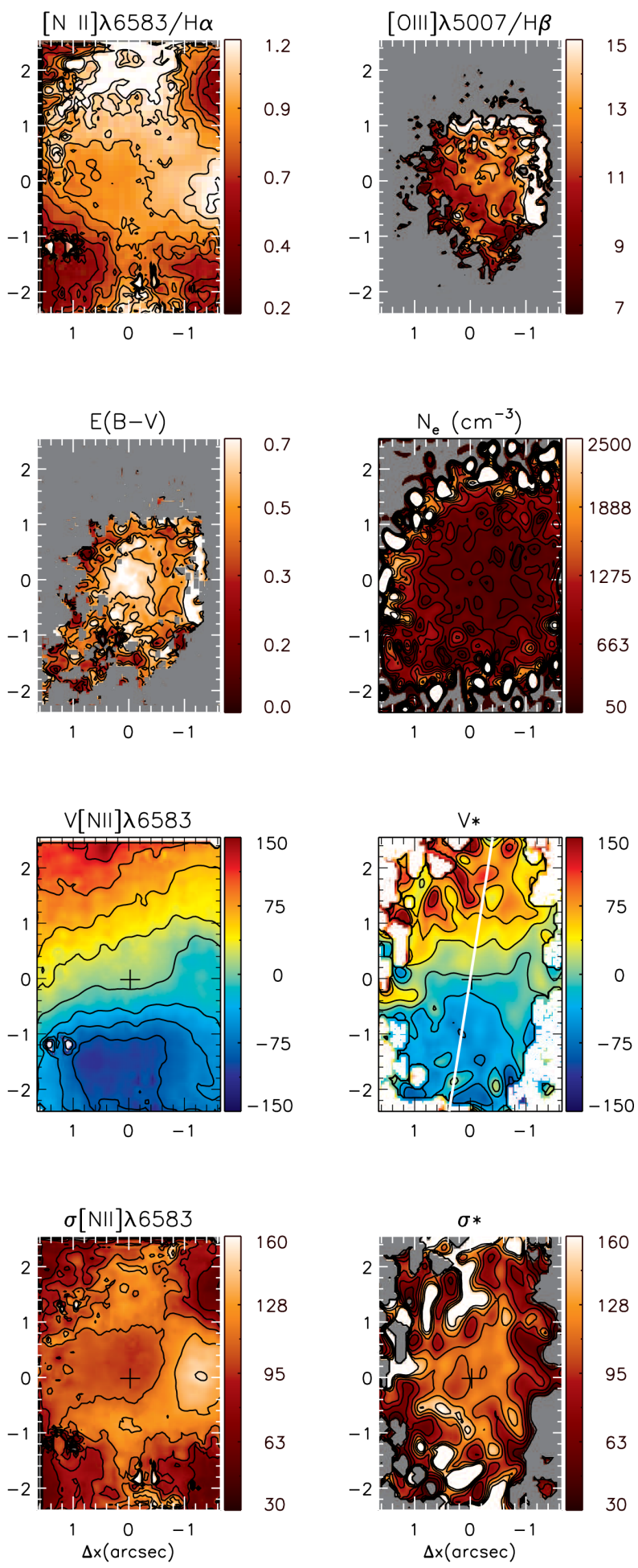

Figure 7. Same as Fig. 4 for Mrk 1058. (xv) Bottom third panel: same as previous panel, for the [ $\left.\mathrm{N}_{\mathrm{II}}\right]$ $\lambda 6583$ emission line;

(xvi) Bottom right panel: same as previous panel, for the stars.

In all maps, the white/grey regions correspond to masked regions where the $\mathrm{S} / \mathrm{N}$ ratio was not high enough to allow good fits of the emission-line profiles. At other locations, the uncertainties in flux are smaller than 30 percent and in velocity/ $\sigma$ smaller than
$30 \mathrm{~km} \mathrm{~s}^{-1}$. For most locations, the uncertainty in the [O $\left.\mathrm{III}\right] \lambda 5007$ and $\mathrm{H} \alpha$ flux and velocities are smaller than 10 percent and 15 $\mathrm{km} \mathrm{s}^{-1}$, respectively.

The emission-line flux distributions for other emission lines, nominally $\mathrm{H} \beta$, [O I ] $\lambda 6300$, [N II] $\lambda 6583$ and [S II] $\lambda 6731$, are shown in Fig. A1 of Appendix A. We do not show the kinematic maps for these lines, as they are similar to that of $\mathrm{H} \alpha$ and [O III] $\lambda 5007$ shown in Figs 3-7. 


\subsection{Emission-line flux distributions}

All galaxies show extended emission up to the borders of the observed field of view (FoV) for the strongest emission lines, corresponding to typical extents of $0.6-1.5 \mathrm{kpc}$ at the galaxies. The lineemission peak is observed at the location of the nucleus for all lines and all galaxies. Mrk 6 shows an elongated structure to 1.5 arcsec $(575 \mathrm{pc})$ north of the nucleus at intermediate flux levels, being more evident for the strongest emission lines $\left(\mathrm{H} \alpha,\left[\mathrm{O}\right.\right.$ III] $\lambda 5007$ and $\left[\mathrm{N}_{\mathrm{II}}\right]$ $\lambda 6583)$, while at low flux levels, the emission is more elongated along the northwest-southeast direction, approximately coincident with the orientation of the major axis of the galaxy as seen in the large-scale image. The elongation to the north is in agreement with that observed in the HST [O III] image, although this latter shows several other sub-structures due to its higher spatial resolution.

For Mrk 79, all lines show similar flux distributions. The flux maps show a curved elongation up to the border of the FoV $1110 \mathrm{pc}$ to the north of the nucleus and a similar elongation to the south. This structure makes an angle of $\approx 50^{\circ}$ with the large-scale disc major axis, as seen in the HST F606W image. This structure is observed in the HST narrow-band image as a much more collimated and with smaller scale features due to the better spatial resolution.

The $\mathrm{H} \beta$ and [O I] $\lambda 6300$ emission for Mrk 348 (Fig. A1) are restricted to the inner $\approx 1.5 \operatorname{arcsec}(465 \mathrm{pc})$, while others maps show extended emission over the whole FoV $(1.1 \mathrm{kpc} \times 1.5 \mathrm{kpc})$, with the highest levels being more elongated along the northeast-southwest direction. The [O III] HST image shows two blobs of higher emission, one centred at the nucleus and another at $\approx 1.0$ arcsec southwest of it.

Mrk 607 shows extended emission over the whole FoV $(0.6 \mathrm{kpc}$ $\times 0.9 \mathrm{kpc})$ for most emission lines. The highest flux levels are more extended along the southeast-northwest direction, in agreement with that observed in the HST [O III] image. The $\mathrm{H} \beta$ and $\left[\mathrm{O}_{\mathrm{I}}\right] \lambda 6300$ emission maps are more compact, with the [O I] one being showing a north-south elongation within the inner $\approx 0.8 \operatorname{arcsec}$ (140 pc).

For Mrk 1058, the highest flux levels for the [O III] emission are more elongated to the southwest, in agreement with the HST [O III] image, that shows a structure there at $1.0 \operatorname{arcsec}(350 \mathrm{pc})$ from the nucleus. The other flux distributions (e.g. $\mathrm{H} \alpha$ ) show also extended emission approximately along the west-east direction up to the borders of the FoV.

\subsection{Line-of-sight velocity maps}

The $V_{\mathrm{LOS}}$ maps for all galaxies are shown in Figs $3-7$. The velocities are shown relative to the systemic velocity of each galaxy, adopted as the value obtained for the stellar velocity within the inner 0.45 arcsec $\times 0.45$ arcsec, with exception of Mrk 6 , for which the adopted value corresponds to the central wavelength of the narrow component of the $\mathrm{H} \beta$ emission line of the nuclear spectra for the same aperture. The derived heliocentric systemic velocities are $5603,6545,4473,2782$, and $5053 \mathrm{~km} \mathrm{~s}^{-1}$ for Mrk 6, Mrk 79, Mrk 348, Mrk 607, and Mrk 1058, respectively.

The $V_{\mathrm{LOS}}$ maps for Mrk 6 (Fig. 3) present a distorted rotation pattern with blueshifts observed to the north of the nucleus and redshifts to the south of it, with a projected velocity amplitude of $\approx 200 \mathrm{~km} \mathrm{~s}^{-1}$. The distortions in the rotation pattern differ between the $\mathrm{H} \alpha$ and $[\mathrm{O}$ III] rotation fields, while the highest redshifts are observed at $\approx 1.0$ arcsec south of the nucleus for the [O III], for $\mathrm{H} \alpha$ the highest velocities are observed next to the corner of the IFU FoV at $\approx 2.0$ arcsec southeast of the nucleus. The [N II] velocity field is similar to that of $\left[\mathrm{O}_{\mathrm{III}}\right]$ and we were not able to map the stellar kinematics for this galaxy, due to the low $\mathrm{S} / \mathrm{N}$ ratio of the stellar absorption features.

The most conspicuous structures observed in the Mrk 79 gas velocity fields are two 'blobs' observed in blueshifts, of up to $-90 \mathrm{~km} \mathrm{~s}^{-1}$, one at $\approx 1.0 \operatorname{arcsec}(444 \mathrm{pc})$ to the north and another at $\approx 1.5 \operatorname{arcsec}(666 \mathrm{pc})$ to the south-southeast of the nucleus, at locations coincident with blobs seem in the HST [O III] image. In addition, some redshifts are observed to west of the nucleus at distances larger than 2.0 arcsec from it, more clearly seen in the $\mathrm{H} \alpha$ $V_{\text {LOS }}$ map. We were able to measure the stellar kinematics only within the inner 1 arcsec and the corresponding stellar $V_{\text {LOS }}$ map show blueshifts to the southeast and redshifts to the north-west, approximately along the orientation of the photometric major axis of the galaxy.

In the case of Mrk 348 (Fig. 5), the main gas kinematics structures are two blobs observed at $\approx 1.5 \operatorname{arcsec}(465 \mathrm{pc})$ to the northeast and southwest of the nucleus, observed in all $V_{\text {LOS }}$ maps. The blob to the northeast shows blueshifts of up to $-200 \mathrm{~km} \mathrm{~s}^{-1}$, while the one to the southwest shows redshifts with similar amplitudes. The emission-line profiles at these locations are very complex and not well reproduced by a single Gaussian component, possible indicating the presence of gas outflows from the galaxy nucleus and suggest a bipolar outflow from the nucleus. The stellar velocity field shows a smaller velocity amplitude of $\sim 50 \mathrm{~km} \mathrm{~s}^{-1}$ with redshifts observed to the east and blueshifts to the west.

A clear rotation pattern is observed for the gas in Mrk 607 (Fig. 6), with the line of nodes oriented along the PA $\approx-60 / 120^{\circ}$, with blueshifts observed to the north-west and redshifts to the southeast. The observed gas velocity amplitude is $\approx 150 \mathrm{~km} \mathrm{~s}^{-1}$. A rotation pattern is also observed for the stars, but at the opposite orientation, with blueshifts seen to the southeast and redshifts to the north-west. In addition, the stellar $V_{\text {LOS }}$ map shows a smaller velocity amplitude of $\sim 70 \mathrm{~km} \mathrm{~s}^{-1}$.

The stellar $V_{\text {LOS }}$ map for Mrk 1058 shows a rotation pattern along the photometric major axis of the galaxy, with blueshifts seen to the southeast and redshift to the north-west, with a projected velocity amplitude of $\sim 120 \mathrm{~km} \mathrm{~s}^{-1}$. We see a distorted rotation pattern in the $\mathrm{H} \alpha$ and $[\mathrm{N}$ II] velocity fields with blueshifts and a steeper rotation pattern to the southeast and redshift to the north-west, and a projected velocity amplitude of $\approx 150 \mathrm{~km} \mathrm{~s}^{-1}$. In the case of [O III], the velocity field seems more disturbed, with blueshifts dominating the velocities. In particular, blueshifts observed at $\approx 1.5 \operatorname{arcsec}(522 \mathrm{pc})$ southwest of the nucleus (less conspicuous but also present in the $\mathrm{H} \alpha$ velocity field) are cospatial with the elongation seen in the [O III] images, suggesting it is an outflow from the nucleus.

\subsection{Velocity dispersion maps}

The gas and stellar velocity dispersion maps for Mrk 6, Mrk 79, Mrk 348, Mrk 607, and Mrk 1058 are shown in the bottom panels of Figs 3-7.

Mrk 6 (Fig. 3) shows $\sigma$ values ranging from $\approx 100$ to $\approx 450 \mathrm{~km} \mathrm{~s}^{-1}$. The highest $\sigma$ values for $\mathrm{H} \alpha$ are seen in a broad arc-shaped region centred at 0.7 arcsec to the north of the nucleus (bottom left panel of Fig. 3). This structure seems to correspond to the extended emission observed in the HST [O III] image. The [O III] $\sigma$ map also shows an arc-shaped structure, partially cospatial with that observed in $\mathrm{H} \alpha$, but the highest values are observed (at similar distances) to the west of the nucleus. The [N II] shows overall smaller values of $\sigma$ than [O III], but with the highest values observed at the same position to the west. 
The gas $\sigma$ maps for Mrk 79 (Fig. 4) show values from 80 to $180 \mathrm{~km} \mathrm{~s}^{-1}$, with the [O III] showing systematically larger values than $\mathrm{H} \alpha$ and $[\mathrm{N}$ II]. This may indicate that the [O III] emitting gas is located at larger latitudes, while the $\mathrm{H} \alpha$ and [N $\mathrm{NI}]$ emission have a more important contribution from gas located close to the plane of the disc.

The highest values are observed in a bipolar structure oriented along the east-west direction, perpendicular to the direction of the strongest $[\mathrm{O} \mathrm{III}]$ and $\mathrm{H} \alpha$ emission. The stars show small $\sigma$ values at all locations, with $\sigma<100 \mathrm{~km} \mathrm{~s}^{-1}$.

The $\sigma$ values for the emission lines of Mrk 348 (Fig. 5) are in the range: $50-200 \mathrm{~km} \mathrm{~s}^{-1}$, with the highest values observed at the blueshifted and redshifted blobs seen in the velocity fields at $\approx 1.5 \operatorname{arcsec} \mathrm{NE}$ and $\approx 1.3 \operatorname{arcsec}$ southwest of the nucleus, respectively. This is consistent with the interpretation that they correspond to outflowing gas. At locations closer to the nucleus, the $\sigma$ values are between 150 and $200 \mathrm{~km} \mathrm{~s}^{-1}$, while the lowest values are observed in regions beyond the blobs. The stellar velocity dispersion map presents values smaller than $150 \mathrm{~km} \mathrm{~s}^{-1}$ at all locations, showing a drop at the nucleus with $\sigma \sim 50 \mathrm{~km} \mathrm{~s}^{-1}$.

Mrk 607 (Fig. 6) shows the lowest average gas $\sigma$ values of our sample, ranging from 50 to $130 \mathrm{~km} \mathrm{~s}^{-1}$. The highest $\sigma$ values are observed in a strip perpendicular to the galaxy major axis, between the nucleus and $\approx 1.3 \operatorname{arcsec}(227 \mathrm{pc})$ to the southwest, opposite (relative to the nucleus) to the main elongation seen in the [O III] flux map. A distinct behaviour is seen at the $\sigma_{*}$ map, that presents the highest values at distances of 1-2 arcsec to the north-west and to the southeast, along the major axis of the galaxy.

For Mrk 1058 (Fig. 7), $\mathrm{H} \alpha$ and [N $\mathrm{II}] \sigma$ maps shows somewhat smaller values than the [O III] map $\left(40-120 \mathrm{~km} \mathrm{~s}^{-1}\right.$ versus $\left.40-150 \mathrm{~km} \mathrm{~s}^{-1}\right)$. The highest values are observed $\approx 1.3 \operatorname{arcsec}$ $(452 \mathrm{pc})$ to the southwest of the nucleus, at the location where excess blueshifts and extended [O III] emission is observed, consistent with the presence of an outflow. A partial ring of intermediate $\sigma$ values seems to surround the nucleus at 1.0 arcsec. This partial ring is not observed in the stellar $\sigma$ map, which shows values smaller than $130 \mathrm{~km} \mathrm{~s}^{-1}$ at most locations.

\subsection{Line ratio maps and diagnostic diagram}

The emission-line ratios $[\mathrm{N}$ II $] \lambda 6583 / \mathrm{H} \alpha$ and $\left[\mathrm{O}_{\mathrm{III}}\right] \lambda 5007 / \mathrm{H} \beta$ are frequently used to map the NLR excitation via the BPT diagram (Baldwin, Phillips \& Terlevich 1981), enabling to distinguish the gas excitation as characteristic of Seyfert, starburst, low-ionization nuclear emission-line region (LINER) or Transition Objects. We have obtained this diagram for the central regions of the galaxies of our sample in Fig. 8, with each spaxel corresponding to a point in the diagram. We included in this plot only regions where the flux uncertainties of the four emission lines are smaller than 30 per cent, in order to avoid spurious features. The value of these ratios for the spaxels of each galaxy are shown as points with distinct colours: Mrk 6 in red, Mrk 79 in yellow, Mrk 348 in green, Mrk 607 in blue, and Mrk 1058 in purple. The dashed curves represent the division lines between star-forming galaxies and AGN from Kauffmann et al. (2003) and Kewley et al. (2001). The solid line shows the division between Seyfert and LINER nuclei (Cid Fernandes et al. 2010).

For the five galaxies, all spaxels are observed in the Seyfert region of the BPT diagram, showing that the gas excitation in the inner kiloparsec of all galaxies of the sample is dominated by radiation from the AGN.

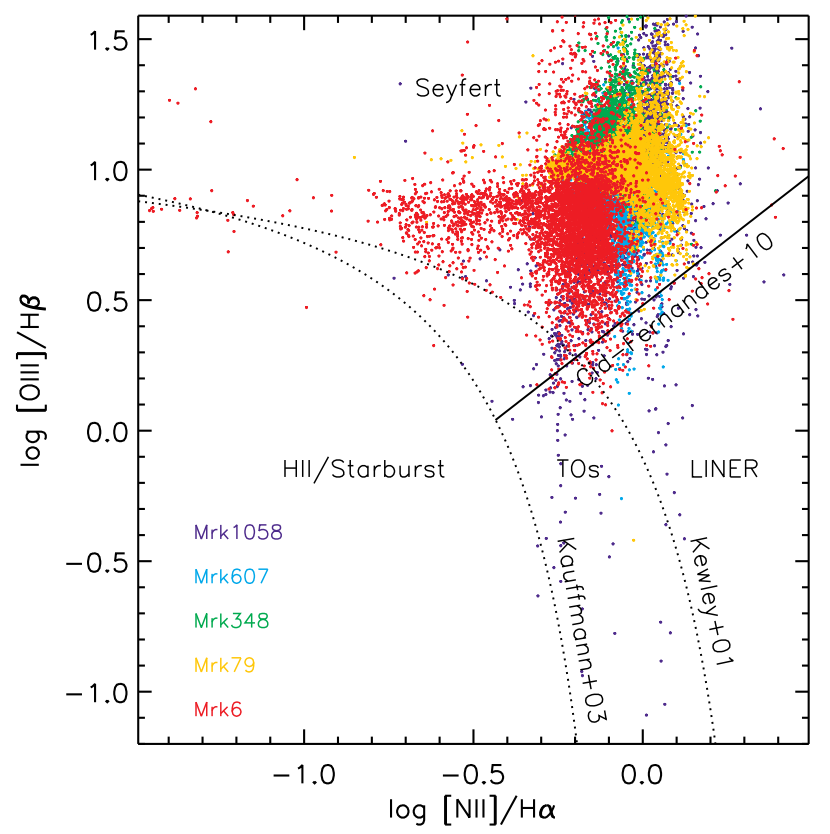

Figure 8. BPT diagram $\left[\mathrm{O}_{\mathrm{III}}\right] \lambda 5007 / \mathrm{H} \beta$ versus $\left[\mathrm{N} \mathrm{II}_{\mathrm{II}}\right] \lambda 6583 / \mathrm{H} \alpha$ line ratios for all the observed galaxies. Each galaxy is represented by a colour.

Although the BPT diagram shows only points within the Seyfert region for all galaxies, spatial variations of the excitation of the NLR can be observed in the $[\mathrm{N}$ II $] / \mathrm{H} \alpha$ and $[\mathrm{O}$ III] $/ \mathrm{H} \beta$ maps of Figs 3-7.

For Mrk 6, the $\left[\mathrm{N}_{\mathrm{II}}\right] / \mathrm{H} \alpha$ map (Fig. 3) shows values ranging from 0.4 to 0.8 , with most values close to the maximum and the lowest values observed mostly to the northeast of the nucleus, approximately cospatial with the arc region showing the highest $\sigma$ values in $\mathrm{H} \alpha$ and also with the highest reddening (see the next section). $[\mathrm{O}$ III $] / \mathrm{H} \beta$ shows values around 8 in an $\approx 1.0 \operatorname{arcsec}$ wide stripe passing through the nucleus from the northeast to the southwest, approximately following the orientation of the HST [O III] image. Lower values down to approximately 0.5 are seeing surrounding the strip, with a few spots with higher values.

The $[\mathrm{N}$ II] $/ \mathrm{H} \alpha$ ratio map for Mrk 79 (Fig. 4) shows the smallest values $(\approx 0.3)$ at the nucleus and increasing outwards, up to 1.3 at distances larger than 2.0 arcsec. The $[\mathrm{O} \mathrm{III}] / \mathrm{H} \beta$ map presents values in the range $4-11$, with values of about 10 approximately along the north-south direction, following the orientation of the collimated [O III] emission observed in the HST narrow-band image, and are surrounded to the east and west by the smallest ratios of the order of 6 . The highest values $(\geq 12)$ are observed to the south at the border of the field and distances of $\geq 1.0$ arcsec.

Mrk 348 (Fig. 5) shows $\left[\mathrm{N}_{I I}\right] / \mathrm{H} \alpha$ ratio ranging from 0.6 to 1.2. The smallest values are observed at approximately $1.5 \operatorname{arcsec}$ to the southwest, at a location just beyond the extended emission observed in the HST [O III] map and the GMOS $\mathrm{H} \alpha$ and [O III] maps. The highest values are observed to the north-northeast of the nucleus, at distances of $\approx 2.0$ arcsec from the nucleus. Due to the low $\mathrm{S} / \mathrm{N}$ ratio of the $\mathrm{H} \beta$ emission line, we were able to obtain the $[\mathrm{O}$ III] $/ \mathrm{H} \beta$ only for the inner 1.5 arcsec. The map has the highest values $(\approx 15)$ to the northeast and the lowest ratios $(\approx 10)$ to the southwest.

Fig. 6 shows that the $[\mathrm{N} I I] / \mathrm{H} \alpha$ map for Mrk 607 presents the lowest values $(0.5-0.7)$ at the nucleus and that this value increases outwards to higher than 1.2 in two 'strips' at $\approx 2.0 \operatorname{arcsec}$ southeast and $\approx 2.0$ arcsec west of the nucleus. The $[\mathrm{O}$ III]/H $\beta$ map shows values ranging from 3 to more than 10 , with the highest ones observed 
at the nucleus and to the north-northeast and east, and the lowest values seen to the north-west.

Mrk 1058 (Fig. 7) shows $[\mathrm{N}$ II] $/ \mathrm{H} \alpha$ ranging from 0.4 to 1.2 , with the smallest values observed to the east, south and west at distances larger than 1.0 arcsec from the nucleus, at locations where the $\mathrm{H} \alpha$ flux distribution presents elongated structures. The highest values for this ratio are seen at $\approx 1.0 \operatorname{arcsec}$ southwest and $\approx 2.0 \operatorname{arcsec}$ north-west of the nucleus, while intermediate values (0.9-1.0) are observed between these locations. The $\left[\mathrm{O}_{\mathrm{III}}\right] / \mathrm{H} \beta$ ratio map presents a gradient along the east-west direction (similar to the orientation of the extended $\mathrm{H} \alpha$ emission), with lowest values $(\approx 9)$ to the east and the highest values $(\approx 15)$ to the west.

\subsection{Reddening maps}

The reddening maps obtained from the $\mathrm{H} \alpha / \mathrm{H} \beta$ line ratio are presented in the top right panels of Figs 3-7. Mrk 6 (Fig. 3) presents $E(B-V)$ values ranging from 0.2 to 0.9 , with the highest values observed from the nucleus up to 1.0 arcsec to the northeast, at the location where an increase in the $\mathrm{H} \alpha$ velocity dispersion is observed.

For Mrk 79 (Fig. 4) the $E(B-V)$ values range from 0.4 to 0.8 with the smallest values close to the nucleus and increasing outwards along the direction of extended emission in [O $\mathrm{III}]$, particularly to the south-southwest at a region of enhanced $[\mathrm{O} I I] / \mathrm{H} \beta$ ratio and blueshifts observed in the $\mathrm{H} \alpha$ and $[\mathrm{O} \mathrm{III}]$ velocity fields.

Mrk 348 (Fig. 5) shows $0.2<E(B-V)<0.7$, with the highest values observed at the nucleus up to $\approx 1.0 \operatorname{arcsec}$ from it along the east-southeast to west-northwest, and at the location where an extension of the [O III] HST emission is observed to the southsouthwest $(\approx 0.8$ arcsec from the nucleus).

The $E(B-V)$ map for Mrk 607 (Fig. 6) shows values in the range $0.2-0.7$, with the highest values observed at the nucleus up to $\approx 1.0$ arcsec to the northeast and the lowest values seen to the opposite side and to the north-west, along the major axis of the galaxy.

Mrk 1058 (Fig. 7) shows $E(B-V)$ smaller than 0.5 at most locations. The highest values of up to 0.7 are observed at the nucleus and within a radius of $\approx 0.4$ arcsec from it.

\subsection{Electron density}

We used the temden IRAF routine to obtain electron density $\left(N_{\mathrm{e}}\right)$ maps using the $[\mathrm{S}$ II] $\lambda \lambda 6716 / 6731$ flux ratio and assuming an electronic temperature $T_{\mathrm{e}}=10000 \mathrm{~K}$. The resulting maps for each galaxy are shown in the bottom right panel of Figs 3-7.

For Mrk 6 (Fig. 3), the $N_{\mathrm{e}}$ map shows values ranging from 100 to $1800 \mathrm{~cm}^{-3}$, with higher values observed within $\approx 1.0$ arcsec from the nucleus along the northeast-southwest direction. The highest values are seen in two blobs located at 0.25 arcsec northeast and 0.45 arcsec southwest of the nucleus. At the nucleus $N_{\mathrm{e}} \approx 1300 \mathrm{~cm}^{-3}$.

The $N_{\mathrm{e}}$ map for Mrk 79 (Fig. 4) shows $100<N_{\mathrm{e}}<900$, with the highest values observed within $\approx 0.4$ arcsec from the nucleus. Some high values are also observed outwards towards the east and west, cospatial regions with enhanced gas velocity dispersion in [O III] $\lambda 5007$ and $\mathrm{H} \alpha$.

Mrk 348 (Fig. 5) shows $N_{\mathrm{e}}$ in the range $100-1100 \mathrm{~cm}^{-3}$, with the highest values observed at the nucleus. The $N_{\mathrm{e}}$ map shows an elongated structure to the southwest where $N_{\mathrm{e}} \approx 850 \mathrm{~cm}^{-3}$ at $1.5 \operatorname{arcsec} \mathrm{SW}$ of the nucleus. This peak is observed cospatially with an enhancement in the velocity dispersion and a redshifted blob in the gas velocity fields.
The electron density map for Mrk 607 (Fig. 6) shows values from 100 to up to $1400 \mathrm{~cm}^{-3}$ with the highest values at the nucleus. An elongated structure with extent $\approx 1.2$ and $0.5 \operatorname{arcsec}$ width with $N_{\mathrm{e}}$ $\approx 1100 \mathrm{~cm}^{-3}$ is observed along the northeast-southwest direction, $\approx$ perpendicularly to the major axis of the galaxy, and along the direction where the highest $[\mathrm{O}$ III] $] / \mathrm{H} \beta$ ratios are observed. Enhancements in the velocity dispersions of both [O III] and $\mathrm{H} \alpha$ are observed contiguous to this structure to the southeast.

Mrk 1058 (Fig. 7) presents the highest values of $N_{\mathrm{e}}$ among the galaxies of our sample, with $N_{\mathrm{e}}$ ranging from 100 to up to $2500 \mathrm{~cm}^{-3}$. The highest values are observed at a ring at with radius of $\approx 1.5$ arcsec, surrounding the nucleus. This ring seems to be correlated with the highest $\sigma$ values seen at the bottom left/central panels of Fig. 7. However, the interpretation of high $N_{\mathrm{e}}$ structures derived from the $\left[\mathrm{S}_{\mathrm{II}}\right]$ lines must be done with caution, as the relation between the line ratio and $N_{\mathrm{e}}$ is rather flat for $N_{\mathrm{e}} \gtrsim 2000 \mathrm{~cm}^{-3}$ (Osterbrock \& Ferland 2006) and thus estimates of $N_{\mathrm{e}}$ in this range could also be originated by uncertainties in the line flux measurements. Within the ring, smaller $N_{\mathrm{e}}$ values are seen, typically smaller than $1000 \mathrm{~cm}^{-3}$.

\section{DISCUSSION}

\subsection{Mrk 6}

Mrk 6 is an S0a galaxy and harbours a compact Seyfert 1.5 nucleus (e.g. Osterbrock \& Koski 1976; Haniff, Wilson \& Ward 1988; Kharb et al. 2006; Mingo et al. 2011). Capetti et al. (1995) presented narrow-band images of the inner $1.4 \operatorname{arcsec} \times 1.4 \operatorname{arcsec}$ of Mrk 6 centred at $\left[\mathrm{O}_{\mathrm{III}}\right] \lambda \lambda 4959,5007$ and $\left[\mathrm{O}_{\mathrm{II}}\right] \lambda \lambda 3726,29$ emission lines, obtained with the Faint Object Camera on board the HST. Their images show a jet-like emission feature to the south/southwest of the nucleus with extent of 0.5 arcsec, which is cospatial with a radio jet seen in $6 \mathrm{~cm}$ Multi-Element Radio-Linked Interferometer Network (MERLIN) observations. The authors argue that these results support the interpretation that the emission is dominated by compression and heating of the gas by shocks produced by the radio jet. On larger scales, ground based [ $\left.\mathrm{O}_{\mathrm{III}}\right] \lambda 5007$ narrowband and long-slit spectroscopy at a seeing of 1.0 arcsec show an extended NLR (eNLR), visible out to 35.0 arcsec from the nucleus with several knots and diffuse emission, mostly to the north and south of the nucleus (Kukula et al. 1996). The authors found that the eNLR is misaligned with the large-scale radio axis, as revealed by the comparison of the [O III] image with 6 and $18 \mathrm{~cm}$ radio images obtained with MERLIN.

A high spatial resolution [O III] $\lambda 5007$ image of Mrk 6, obtained with the WFPC2 of HST (shown in Fig. 3, Schmitt et al. 2003) confirms the jet-like structure to the north of the nucleus (along $\left.\mathrm{PA}=-10^{\circ}\right)$, misaligned with the major axis of the galaxy ( $\mathrm{PA}=$ $\left.130^{\circ}\right)$. The peak of the $[\mathrm{O} \mathrm{III}]$ emission is observed at the nucleus and several blobs are observed around it and a fainter emission is observed along the major axis of the galaxy (Schmitt et al. 2003).

Very Large Array (VLA) observations of Mrk 6 show radio emission at different spatial scales, with bubbles observed at $\approx 7.5 \mathrm{kpc}$, nearly orthogonal to the inner jet that extends by approximately 3.0 arcsec in the north-south direction. This complex radio emission is argued to have been possibly originated by an episodically powered precessing jet that changes its orientation (Kharb et al. 2006). The inner jet - as observed at $3.6 \mathrm{~cm}$ (Schmitt et al. 2001) - is shown overplotted in our [O III] flux map as green contours in Fig. 3. X-ray observations of Mrk 6 reveal shells of X-ray emission around the radio hotspots, with a temperature of $\approx 0.9 \mathrm{keV}$, 
compatible with a scenario in which the gas in the shells is inducing a strong shock in the interstellar medium (Mingo et al. 2011).

Quillen et al. (1999) present broad (F160W) and narrow (centred at the $\mathrm{H}_{2} \lambda 2.12 \mu \mathrm{m}$ emission line) band images obtained with the Near-Infrared Camera and Multi object Spectrometer on board the HST. The narrow-band image does not show extended molecular hydrogen emission, attributed the nuclear source of Mrk 6 being so bright that it is difficult to observe any extended structure.

The mass of the central SMBH is about $(1-2) \times 10^{8} \mathrm{M}_{\odot}$ as estimated by Keplerian motion in the BLR using spectropolarimetry (Afanasiev et al. 2014) and from measurements of the $\mathrm{H} \beta$ line width in combination with the reverberation lag (Doroshenko et al. 2012).

Our emission-line flux distributions (Fig. 3) do not clearly show the jet-like compact inner emission seen in the HST image in the north-south direction due to the effect of the seeing, showing more the diffuse emission that extends to all directions. The gas kinematics shows a rotation pattern with somewhat distinct orientation in $\mathrm{H} \alpha$ and [O III]. An increase of the velocity dispersion is observed to the north in $\mathrm{H} \alpha$ reaching $\sigma=450 \mathrm{~km} \mathrm{~s}^{-1}$, apparently at the tip of the nuclear jet, that could then be attributed to compression by the jet. The increase in $\sigma$ is lower in [O $\mathrm{III}]$ and [N $\mathrm{NI}$, and a possible explanation is that there is some radiation ionizing $\mathrm{H}$ in the plane, and this gas is compressed by the radio jet that is launched close to the plane. But the ionization axis would make an angle with the galaxy plane and higher ionization (higher $[\mathrm{O} \mathrm{III}] / \mathrm{H} \beta$ ) would thus be observed at higher latitudes, being less affected by the compression by the radio jet. The highest $E(B-V)$ values seen at the same location could also be the result of the accumulation of dust due to compression by the radio jet. In [O III], there is also enhanced $\sigma$ to the west, what could be attributed to lateral expansion of the gas due to the passage of the radio jet. These results can be interpreted as an evidence of the interaction of gas outflows from the central AGN with surrounding gas in the galaxy, allowing the detection of emission from gas located deep in the galaxy disc (with higher extinction), and producing distortions in the gas velocity fields.

\subsection{Mrk 79}

Mrk 79 (UGC 3973) is an SBb galaxy harbouring a Seyfert 1.2 nucleus (e.g. Haniff et al. 1988; de Vaucouleurs et al. 1991; Malkan et al. 1998; Kraemer et al. 2011). Peterson et al. (2004) determined a mass of $52.4 \pm 14.4 \times 10^{6} \mathrm{M}_{\odot}$ for the central SMBH, through BLR emission-line reverberation.

Ground-based (Haniff et al. 1988) and HST (top central panel of Fig. 4, Schmitt et al. 2003) narrow-band images in the [O III] $\lambda 5007$ emission line show a collimated emission oriented along north-south direction, extending up to $\approx 3.0$ arcsec to the north and $\approx 2.0$ arcsec to the south of the nucleus. Close to the northern edge, it bends to the northeast. Fainter emission is observed over the whole $H S T$ FoV of 8.0 arcsec $\times 8.0$ arcsec. Schmitt et al. (2003) describe the $[\mathrm{O} \mathrm{III}]$ image as presenting two blobs of emission at 0.6 and 1.2 arcsec south of the nucleus. To the north, blobs are seen at $0.5,0.9$, and 1.6 arcsec from the nucleus. Considering the lower spatial resolution and lower spatial coverage of the GMOS data, as compared to the HST, our flux maps (Figs 4 and A1) for all emission lines are consistent with the HST image, showing a similar elongated structure along the north-south direction, with the northern side bending to the northeast and fainter emission seen over the whole GMOS FoV.

Long-slit spectra of Mrk 79, obtained with the William Herschel Telescope, covering the wavelength ranges 3700-5230 ̊ and 6110 $-7460 \AA$, oriented along $\mathrm{PA}=12^{\circ}$ and $50^{\circ}$, reveal an eNLR at
$\mathrm{PA}=12^{\circ}$, extending up to $\approx 15.0$ arcsec from the nucleus (Nazarova, O'Brien \& Ward 1996). The eNLR approximately follows an asymmetric triple radio structure - with the northern hotspot located at a distance of $800 \mathrm{pc}$ from the nucleus and the southern at $460 \mathrm{pc}$ from it (Ulvestad \& Wilson 1984; Nagar et al. 1999; Schmitt et al. 2001). This is also the direction of a gas outflow, suggested by multicomponents $[\mathrm{O}$ III] $\lambda 5007$ profiles along the radio axis (Whittle et al. 1988). We have overplotted contours of the radio structure as observed in $3.6 \mathrm{~cm}$ from Schmitt et al. (2001), showing that it indeed is oriented along the extended emission observed in $\mathrm{H} \alpha$ and [O III].

Riffel et al. (2013) observed the inner $3 \operatorname{arcsec} \times 3 \operatorname{arcsec}$ of Mrk 79 with the Gemini instrument Near-infrared Integral Field Spectrograph (NIFS) showing that the near-IR ionized gas emission presents a similar flux distribution to those seen in optical wavelengths. The $\mathrm{H}_{2} \lambda 2.12 \mu \mathrm{m}$ shows, on the other hand, a more uniform flux distribution and a rotation pattern with the north-west side receding and the southeast side approaching; in addition, inflows of gas are seen along spiral arms. The ionized gas (traced by [Fe II] $\lambda 1.25 \mu \mathrm{m}$ and $\mathrm{P} \beta$ emission) shows, besides rotation, an outflow seen as blueshifts to the north-northeast and redshifts to the opposite side. Our velocity fields are consistent with those for the ionized gas in Riffel et al. (2013): (i) we also see a rotation component - redshifts to the north-west, close to the border of the FoV, and blueshifts to the southeast. Additional support to the presence of this rotation component is given by the stellar velocity filed, that shows blueshifts and redshifts at the same locations; (ii) we also see an outflowing component - blueshifts to the north-northeast and some (less clear) redshifts to the south-southwest, along the same orientation $\left(\mathrm{PA} \approx 10^{\circ}\right.$ ) of the outflows seen in the near-IR lines (Riffel et al. 2013) and at larger scales in [O III] (Whittle et al. 1988).

We note that the outflow and strongest gas emission follows the radio structure along $\mathrm{PA}=12^{\circ}$ (what we could call 'ionization axis'). One interesting result that can be seen in our data is the fact that the gas velocity dispersion is largest perpendicularly to this axis, instead of along the direction of the outflow, a feature we have also observed in other targets (e.g. Su et al. 1996; Couto et al. 2013; Riffel, Storchi-Bergmann \& Riffel 2014, 2015; SchnorrMüller et al. 2014b; Lena et al. 2015) and that could be interpreted as due to lateral expansion of the gas due to the passage of a radio jet and/or expansion of the dusty torus surrounding the nucleus.

Regarding the gas excitation, Nazarova et al. (1996) point out the observation of higher excitation along $\mathrm{PA}=12^{\circ}$, when compared to that along $\mathrm{PA}=50^{\circ}$ in long-slit spectroscopic observations along these two PAs, consistent also with our data.

\subsection{Mrk 348}

Mrk 348 (NGC 262) is an S0/a galaxy that harbours a bright Seyfert 2 nucleus (Antón et al. 2002). At small scales, Very Long Baseline Interferometry observations at $21 \mathrm{~cm}$ show a compact triple radio structure along $\mathrm{PA} \approx 170^{\circ}$, with total size of only $0.2 \operatorname{arcsec}$ (e.g. Neff \& de Bruyn 1983), while at $2 \mathrm{~cm}$ a small-scale double source is seen with similar extent (Ulvestad et al. 1999). VLA radio images at 3.6 and $20 \mathrm{~cm}$ show an unresolved nuclear radio source and a faint extended emission to up to 4.0 arcsec from the nucleus to the north-northeast and south-southwest, seen at $20 \mathrm{~cm}$ (Nagar et al. 1999). Falcke et al. (2000) report the detection of strong $\mathrm{H}_{2} \mathrm{O}$ maser emission with luminosity of $420 \mathrm{~L}_{\odot}$.

Early ground-based, low-resolution narrow-band images in [O III] and $[\mathrm{N}$ II] $\mathrm{H} \alpha$ emission suggest the presence of highly ionized region extending up to 15.0 arcsec from the nucleus along the north- 
south direction, the same orientation of the small-scale radio axis (Simpson et al. 1996). Higher resolution HST images of the inner 7 arcsec $\times 7$ arcsec also show extended [O III] emission (Capetti et al. 1996; Capetti 2002). The WFPC2 [O III] narrow-band image (Fig. 5, Schmitt et al. 2003) shows extended emission up to $\approx 3.0$ arcsec from the nucleus along $\mathrm{PA} \approx 185^{\circ}$, similarly to that of the $20 \mathrm{~cm}$ radio emission (Nagar et al. 1999). Very close to the nucleus, the emission is extended along $\mathrm{PA} \approx 10^{\circ}$, similar to the orientation of the small-scale radio jet (Neff \& de Bruyn 1983; Ulvestad et al. 1999). Our flux maps for all emission lines (Figs 5 and A1) show extended emission over the whole GMOS FoV with the high intensity flux levels being more extended along the northeast-southwest direction, in agreement with the HST [O III] image. Our electron density map shows two blobs of highest density $\left(N_{\mathrm{e}} \approx 1000 \mathrm{~cm}^{-3}\right)$ that are cospatial with the highest level emission at the nucleus and to $\approx 1.0$ arcsec to the southwest.

Fischer et al. (2013) used HST STIS spectra of a sample of 53 nearby Seyfert galaxies, including Mrk 348, to study the kinematics of the NLR. They classify the [O III] kinematics of Mrk 348 as 'compact', as their slit position missed the extended part of the NLR. Stoklasová et al. (2009) presented optical IFS of the inner $\approx 10 \operatorname{arcsec} \times 8$ arcsec obtained with the OASIS spectrograph at Canada-France-Hawaii Telescope at an angular sampling of 0.27 arcsec. Their $[\mathrm{O} I I] / \mathrm{H} \beta$ map presents higher values at the nucleus (up to 12), decreasing with the distance from the nucleus down to $\approx 0.5$ at $3-40.27$ arcsec east of it, while the $[\mathrm{N}$ II] $/ \mathrm{H} \alpha$ map shows a flat distribution of values of $\approx 1$, with a minimum seen southwest of the nucleus. Although the range of values are similar, our line ratio maps (Fig. 5) reveal more details due to the better spatial resolution of the GMOS data. The $\left[\mathrm{O}_{\mathrm{III}}\right] / \mathrm{H} \beta$ map shows a gradient of higher ionization to the northeast of the nucleus and lower ionization to the southwest. A similar trend is seen for the $\left[\mathrm{N}_{\mathrm{II}}\right] / \mathrm{H} \alpha$ ratio, with the lowest values at $\approx 1.0$ arcsec southwest of the nucleus, at the edge of the [O III] blob seen in the HST image at $\approx 1.0 \operatorname{arcsec}$ to the southeast. Our $E(B-V)$ map shows the highest values of up to 0.7 at the nucleus.

The gas velocity fields in Stoklasová et al. (2009), show two high velocity regions with diameters $\approx 200 \mathrm{pc}$ and opposite velocity signs along $\mathrm{PA}=25^{\circ}$ at distances of $300 \mathrm{pc}$ from the nucleus, similarly to those observed in our velocity fields (Fig. 5), with velocities of up to $200 \mathrm{~km} \mathrm{~s}^{-1}$. These high velocity blobs are also associated with higher velocity dispersion values, of up to $200 \mathrm{~km} \mathrm{~s}^{-1}$. Stoklasová et al. (2009) interpreted these kinematics structures as corresponding to a rotating ring, inclined with respect to the galactic disc. Our interpretation is that these two blobs originate from a nuclear outflow, due to the observation of associated higher velocity dispersion as well as to the fact that they are seen at the same orientation of the extended radio emission (Nagar et al. 1999), and could thus be originated in a jet-cloud interaction. The stellar velocity field suggests a kinematic major axis approximately aligned with the photometric major axis, being almost perpendicular to the ionization axis and nuclear outflow.

\subsection{Mrk 607}

Mrk 607 (NGC 1320) is an Sa nearly edge-on spiral galaxy hosting a Seyfert 2 nucleus (e.g. Mulchaey, Wilson \& Tsvetanov 1996; Ferruit, Wilson \& Mulchaey 2000; Tsai \& Hwang 2015), with an SMBH of mass $(5.5 \pm 2.5) \times 10^{6} \mathrm{M}_{\odot}$, as derived from water maser observations (Gao et al. 2017). Radio images at 3.6, 6, and $20 \mathrm{~cm}$ show a compact core with faint emission extending to the south of the nucleus, being marginally resolved in VLA observations
(Colbert et al. 1996; Nagar et al. 1999; Mundell et al. 2009). A weak radio extension is also seen to the north and north-west of the nucleus at $6 \mathrm{~cm}$ (Colbert et al. 1996).

The [O III] $\lambda 5007$ HST image (top central panel of Fig. 6, Schmitt et al. 2003) shows extended emission by 3.75 arcsec along the major axis of the galaxy $\left(\mathrm{PA}=137^{\circ}\right)$, with the highest intensity levels seen at the nucleus and to the north-west. Along the minor axis the [O III] emission extends by 1.35 arcsec. A similar flux distribution is seen in an [N II] H $\alpha$ HST image (Ferruit 2000). Large-scale narrowband images for the $\mathrm{H} \alpha[\mathrm{N} \mathrm{II}]$ and $[\mathrm{O}$ III] $\lambda 5007$ lines show extended emission up to $\approx 15.0$ arcsec from the nucleus along the major axis of the galaxy (Mulchaey et al. 1996). Our flux maps (Figs 6 and A1) show emission over the whole GMOS-IFU FoV, with the highest intensity levels along the major axis of the galaxy with some enhancement towards the northeast (where the extended HST [O III] emission is mostly observed) in agreement with previous [O III] and $\mathrm{H} \alpha$ [N II] images (Mulchaey et al. 1996; Schmitt et al. 2003; Ferruit 2000).

Ferruit (2000) present an $[\mathrm{O} \mathrm{III}] \lambda 5007 /(\mathrm{H} \alpha[\mathrm{N}$ II] $)$ ratio map, which shows the highest values at the nucleus and smaller values to the north-west. This map can be compared with our [O III] $\lambda 5007 / \mathrm{H} \beta$ (Fig. 6) map, which shows high values of up to 10 at the nucleus and to the northeast, decreasing a bit to the south, and smaller values to the north-west, as observed by Ferruit (2000).

Our $E(B-V)$ map for Mrk 607 shows low values to the northwest, indicating that the collimated line emission seen at this orientation arises from a low-extinction gas. In addition, a trend of higher $E(B-V)$ values to the northeast and lower values to the southwest, along the minor axis of the galaxy is in agreement with large-scale $B-I$ colour maps of Mrk 607, that show higher extinction to the northeast of the nucleus, indicating that the northeast is the near side of the galaxy disc (Kotilainen 1998).

The gas velocity fields (Fig. 6) suggest ordered rotation with the north-west side approaching and the southeast side receding. The stars, on the other hand, rotate in the opposite direction, with blueshifts to the southeast and redshifts to the north-west, in good agreement with the stellar velocity field presented in Riffel et al. (2017), derived from the fitting of the $K$-band CO absorption band heads using Gemini NIFS spectra. The opposite directions of the gas and stars rotation may be related to gravitational interaction of Mrk 607 and its companion NGC 1321 (e.g. Hunt et al. 1999). The $\sigma$ maps for the gas show the highest values of up to $130 \mathrm{~km} \mathrm{~s}^{-1}$ approximately along the minor axis of the galaxy, while the stellar $\sigma$ map presents predominantly small values $\left(\approx 50 \mathrm{~km} \mathrm{~s}^{-1}\right)$ at these locations. As the ionization axis seems to be perpendicular to these structures of high gas $\sigma$, one possibility is that they are tracing equatorial outflows as observed for other active galaxies (e.g. Couto et al. 2013; Schnorr-Müller et al. 2014b; Riffel et al. 2014; Lena et al. 2015), giving support to models of equatorial accretion disc winds (Li, Ostriker \& Sunyaev 2013) and outflowing torus (Hönig et al. 2013; Elitzur 2012; Ivezić \& Elitzur 2010; Mor, Netzer \& Elitzur 2009; Nenkova et al. 2008; Elitzur \& Shlosman 2006). The origin of these high gas $\sigma$ structures cannot be attributed to gas located in the disc, as the stellar velocity dispersion maps present small values at these locations. However, a detailed analysis of the gas kinematics is needed to get a final answer on the origin of these high $\sigma$ structures.

\subsection{Mrk 1058}

Mrk 1058 is an isolated spiral galaxy, classified as Sb and although it harbours a bright Seyfert 2 nucleus (e.g. Corwin, Buta \& de 
Vaucouleurs 1994; Malkan et al. 1998; Chapelon, Contini \& Davoust 1999; Smirnova, Moiseev \& Afanasiev 2010), it lacks detailed studies in the literature. Broad-band HST images reveal dust lanes to the north of the nucleus, suggesting this is the near side of the galaxy (Malkan et al. 1998).

According to De Robertis \& Osterbrock (1986), an integrated nuclear spectrum (aperture 2.7 arcsec $\times 4.0$ arcsec) shows weak emission in $\mathrm{HI}_{\mathrm{I}}$ and $\mathrm{He}_{\mathrm{II}}$ recombination lines and [O I]. They reported also that the $\left[\mathrm{N}_{\mathrm{II}}\right]$ lines are strong relative to $\mathrm{H} \alpha$, a result confirmed by our BPT diagram (Fig. 8) that shows that Mrk 1058 presents the highest average values of $[\mathrm{N} \mathrm{II}] \lambda 6583 / \mathrm{H} \alpha$ of our sample. In addition, the $[\mathrm{O} I I I] \lambda 5007 / \mathrm{H} \beta$ intensity ratio presents high values at all locations.

Mrk 1058 presents only a faint nuclear radio emission at $3.6 \mathrm{~cm}$, with no detected extended emission in VLA observations (Kinney et al. 2000; Schmitt et al. 2001). The HST [O III] 5007 image (Fig. 7 , Schmitt et al. 2003) shows extended emission to up to 2.0 arcsec to the southwest, with a V-shaped morphology with opening angle of $55^{\circ}$ with central axis oriented along $\mathrm{PA}=205^{\circ}$, perpendicular to the major axis of the galaxy (Schmitt et al. 2003). Our [O III] flux map shows emission over the whole GMOS-IFU FoV, but enhanced emission is indeed observed towards the southwest, although not resolving the structure seen in the HST image due to the lower resolution.

The $\mathrm{H} \alpha$ flux distribution is somewhat distinct from that of [O III] showing extended emission to the north-west and southeast of the nucleus. At the edge of these structures (at $\approx 2.0$ arcsec northeast and $\approx 2.0 \operatorname{arcsec}$ southwest), smaller values of $[\mathrm{N}$ II $] / \mathrm{H}_{\alpha}$ are observed. One possible interpretation is the presence of regions of star formation at these locations. Due to the low intensity (or nondetection) of the $\mathrm{H} \beta$ line, we were able to construct the $[\mathrm{O}$ III] $/ \mathrm{H} \beta$ map for these regions only for the inner $1.5 \operatorname{arcsec}$ radius. In this region, there is an ionization gradient, with highest values to the west and lowest to the east.

As for the flux distributions, the kinematics for $\mathrm{H} \alpha$ and [O III] are also distinct from each other, with the $\mathrm{H} \alpha$ velocity field showing a distorted rotation pattern while in the case of [O $\mathrm{III}]$, this pattern is less clear. Both the $\mathrm{H} \alpha$ and [O III] velocity fields seem to show additional blueshifts to the southwest, coincident with the elongated emission seen in the HST [O III] image. The velocity dispersion maps show also enhanced values coincident with the blueshifted region. The stellar velocity field also shows a somewhat similar rotation pattern to that of the gas but does not show the blueshift to the southwest. Considering all these characteristics, combined with the ionization gradient discussed above, we interpret these blueshifts as being originated in gas outflowing from the nucleus.

One particular characteristic of this galaxy is the enhancement of gas density towards the borders of the field at about 1.7 arcsec from the nucleus, that seems to coincide with regions of enhanced velocity dispersion in $[\mathrm{O}$ III]. We speculate that these enhancements are produced by the outflow pushing the surrounding medium, but the uncertainties in $N_{\mathrm{e}}$ may be high at these locations as the relation between the $\left[\mathrm{S}_{\mathrm{II}}\right]$ line ratio and $N_{\mathrm{e}}$ is rather flat for such high values (Osterbrock \& Ferland 2006) and thus high $N_{\mathrm{e}}$ values could also be produced by uncertainties in the $\left[\mathrm{S}_{\mathrm{II}}\right]$ line flux measurements.

\section{CONCLUSIONS}

We have used GMOS-IFU observations covering the wavelength range from 4300 to $7100 \AA$ to map the emission-line flux distributions and kinematics, as well as the stellar kinematics, of the inner kiloparsec of five nearby Seyfert galaxies - Mrk 6, 79, 348, 607, and 1058 - at a spatial resolutions ranging from 110 to $280 \mathrm{pc}$. In this first paper, we present gas flux, excitation, and kinematic maps, reddening and density maps, as well as stellar kinematic maps. In a forthcoming paper, we will use these measurements to obtain gas masses, to model the gas velocity fields in more detail, and to estimate mass flow rates.

The main results of this paper are:

(i) Extended emission over the whole GMOS-IFU FoV ( $3.5 \operatorname{arcsec} \times 5.0 \operatorname{arcsec}$, corresponding to the inner $1-3 \mathrm{kpc}$ at the galaxies) is observed for the strongest emission lines: $\mathrm{H} \beta,\left[\mathrm{O}_{\mathrm{II}}\right] \lambda 5007, \mathrm{H} \alpha,[\mathrm{N}$ II] $\lambda 6583$, and [S $\mathrm{II}] \lambda \lambda 6716,31$. The $[\mathrm{O}$ III $] \lambda 5007 / \mathrm{H} \beta$ versus $[\mathrm{N}$ II] $\lambda 6583 / \mathrm{H} \alpha$ diagnostic diagram is consistent with the line emission being originated from gas excited by the central AGN for all galaxies, although gradients of these line ratios are observed in flux-ratio maps.

(ii) The highest reddening $(E(B-V) \approx 1)$ is usually observed within the inner few hundred parsecs around the nucleus and/or at regions with the highest excitation.

(iii) The highest gas densities $\left(N_{\mathrm{e}} \approx 1000-2000 \mathrm{~cm}^{-3}\right)$ are usually observed at the nucleus, in a few cases also extending towards regions of highest excitation. A particular case is Mrk 1058 that seems to show a circumnuclear ring of high-density gas at $\approx 1.7 \operatorname{arcsec}(592 \mathrm{pc}$ ) from the nucleus.

(iv) The gas kinematics show a distorted rotation pattern that can be attributed to a combination of emission from gas in rotation in the galaxy plane and outflows.

(v) The rotation component of the gas is confirmed by their observation in the stellar kinematics, except for Mrk 607 in which the gas is counter-rotating relative to the stars.

(vi) The gas velocity dispersion shows two typical patterns: it is enhanced at the location of the outflows or at the nucleus perpendicularly to the outflow. This latter behaviour has been attributed to an equatorial outflow at the AGN, possibly originating in the torus.

(vii) The (projected) velocities of the outflow reach at most $\approx 200 \mathrm{~km} \mathrm{~s}^{-1}$, but could be larger as they seem to be mostly in the plane of the sky. The apparent geometries of the outflows can be described as follows:

(1) Mrk 6 shows enhanced gas $\sigma$ values surrounding the radio jet and distortions in the velocity field interpreted as due to an outflow along the jet, but the geometry needs further constraints from modelling.

(2) Mrk 79 shows a bipolar outflow oriented along the ionization axis inferred from the HST [O III] image; another interesting feature is enhanced velocity dispersion in a nuclear strip perpendicular to the outflow that we interpret as due to lateral expansion produced by the passage of the radio jet.

(3) Mrk 348 shows a very clear bipolar outflow in two blobs with opposite velocities relative to the nucleus and enhanced velocity dispersion.

(4) Mrk 607 is seen close to edge on, with the ionization axis along the galaxy plane, and shows distortions in the velocity field that could be due to an outflow that needs further constraints from modelling; enhanced velocity dispersion perpendicular to the inferred axis of the ionization cone and radio axis could be interpreted as lateral expansion of the gas as in the case of Mrk 79.

(5) Mrk 1058, besides presenting a rotation pattern seen in $\mathrm{H} \alpha$ shows also blueshifts mostly in [O III] in association with enhanced velocity dispersion and extended [O III] emission that can be interpreted as the blueshifted part of a nuclear outflow.

In summary, we have found signature of outflows in all galaxies, but their geometry, intrinsic velocities, and resulting mass flow rates 
will be presented in a forthcoming study based on multicomponents fits to the emission lines.

\section{ACKNOWLEDGEMENTS}

We thank an anonymous referee for his/her thorough review, comments and suggestions, which helped us to significantly improve this paper. Based on observations obtained at the Gemini Observatory, which is operated by the Association of Universities for Research in Astronomy, Inc., under a cooperative agreement with the NSF on behalf of the Gemini partnership: the National Science Foundation (United States), the National Research Council (Canada), CONICYT (Chile), Ministerio de Ciencia, Tecnología e Innovación Productiva (Argentina), and Ministério da Ciência, Tecnologia e Inovação (Brazil). ICF thanks the financial support received from CAPES. RAR acknowledges support from FAPERGS and $\mathrm{CNPq}$.

\section{REFERENCES}

Afanasiev V. L., Popović L. Č., Shapovalova A. I., Borisov N. V., Ilić D., 2014, MNRAS, 440, 519

Antón S., Thean A. H. C., Pedlar A., Browne I. W. A., 2002, MNRAS, 336, 319

Antonucci R., 1993, ARA\&A, 31, 473

Bae H.-J., Woo J.-H., Karouzos M., Gallo E., Flohic H., Shen Y., Yoon S.-J., 2017, ApJ, 837, 91

Baldwin J. A., Phillips M. M., Terlevich R., 1981, PASP, 93, 5

Barbosa F. K. B., Storchi-Bergmann T., Cid Fernandes R., Winge C., Schmitt H., 2009, MNRAS, 396, 2

Bruzual G., Charlot S., 2003, MNRAS, 344, 1000

Capetti A., 2002, Rev. Mex. Astron. Astrofis. Conf. Ser., 13, 163

Capetti A., Axon D. J., Kukula M., Macchetto F., Pedlar A., Sparks W. B., Boksenberg A., 1995, ApJ, 454, 85

Capetti A., Axon D. J., Macchetto F., Sparks W. B., Boksenberg A., 1996, ApJ, 469, 554

Cappellari, Emsellem, 2004, PASP, 116, 138

Cardelli J. A., Clayton G. C., Mathis J. S., 1989, ApJ, 345, 245

Chapelon S., Contini T., Davoust E., 1999, A\&A, 345, 81

Cid Fernandes R., Stasińska G., Schlickmann M. S., Mateus A., Vale Asari N., Schoenell W., Sodré L., Jr, 2010, MNRAS, 403, 1036

Ciotti L., Ostriker J. P., Ostriker D., 2010, ApJ, 717, 708

Colbert E. J. M., Baum S. A., Gallimore J. F., O’Dea C. P., Christensen J. A., 1996, ApJ, 467, 551

Corwin H. G., Buta R. J., Jr, de Vaucouleurs G., 1994, AJ, 108, 2128

Couto G. S., Storchi-Bergmann T., Axon D. J., Robinson A., Kharb P., Riffel R. A., 2013, MNRAS, 435, 2982

Crenshaw D. M., Kraemer S. B., Schmitt H. R., Jaffé Y. L., Deo R. P., Collins N. R., Fischer T. C., 2010, AJ, 139, 871

da Silva P., Steiner J. E., Menezes R. B., 2017, MNRAS, 470, 3850

Das V., Crenshaw D. M., Kraemer S. B., Deo R. P., 2006, AJ, 132, 620

Davies R. I. et al., 2014, ApJ, 792, 101

de Grijp M. H. K., Keel W. C., Miley G. K., Goudfrooij P., Lub J., 1992, A\&AS, 96, 389

De Robertis M. M., Osterbrock D. E., 1986, ApJ, 301, 727

de Vaucouleurs G., de Vaucouleurs A., Corwin H. R., Jr, Buta R. J., Pature G., Fouqué P., 1991, Third Reference Catalogue of Bright Galaxies. Springer-Verlag, New York

Diniz M. R., Riffel R. A., Stochi-Bergmann T., Winge C., 2015, MNRAS, 453,1727

Doroshenko V. T., Sergeev S. G., Klimanov S. A., Pronik V. I., Efimov Y. S., 2012, MNRAS, 426, 416

Edmunds M. G., Taylor K., Turtle A. J., 1988, MNRAS, 234, 155

Elitzur M., 2012, ApJ, 747, L33

Elitzur M., Shlosman I., 2006, ApJ, 648, L101

Elvis M., 2000, ApJ, 545, 63
Fabian A. C., 2012, ARA\&A, 50, 455

Falcke H., Henkel C., Peck A. B., Hagiwara Y., Prieto M. A, Gallimore J. F., 2000, A\&A, 358, L17

Ferrarese L., Ford H., 2005, Space Sci. Rev., 116, 523

Ferruit P., Wilson A. S., Mulchaey J., 2000, ApJS, 128, 139

Fischer T. C., Crenshaw D. M., Kraemer S. B., Schmitt H. R., 2013, ApJS, 209, 1

Fischer T. C. et al., 2017, ApJ, 834, 30

Gao F. et al., 2017, ApJ, 834, 52

Haniff C. A., Wilson A. S., Ward M. J., 1988, ApJ, 334, 104

Harrison C. M., Alexander D. M., Mullaney J. R., Swinbank A. M., 2014 MNRAS, 441, 3306

Hönig S. F. et al., 2013, ApJ, 771, 87

Hopkins P. F., Quataert E., 2010, MNRAS, 407, 1529

Hunt L. K., Malkan M. A., Rush B., Bicay M. D., Nelson B. O., Stanga R. M., Webb W., 1999, ApJS, 125, 349

Ivezić Z., Elitzur M., 2010, MNRAS, 404, 1415

Kaspi S., Maoz D., Netzer H., Peterson B. M., Vestergaard M., Jannuzi B. T., 2005, ApJ, 629, 61

Kauffmann G. et al., 2003, MNRAS, 346, 1055

Kewley L. J., Heisler C. A., Dopita M. A., Lumsden S., 2001, ApJS, 132, 37

Kharb P., O'Dea C. P., Baum S. A., Colbert E. J. M., Xu C., 2006, ApJ, 652, 177

Kinney A. L., Schmitt H. R., Clarke C. J., Pringle J. E., Ulvestad J. S., Antonucci R. R. J., 2000, ApJ, 537, 152

Kormendy J., Ho L. C., 2013, ARA\&A, 51, 511

Kotilainen J. K., 1998, A\&AS, 132, 197

Kraemer S. B., Schmitt H. R., Crenshaw D. M., Meléndez M., Turner T. J., Guainazzi M., Mushotzky R. F., 2011, ApJ, 727, 130

Kukula M. J., Holloway A. J., Pedlar A., Meaburn J., Lopez J. A., Axon D. J., Schilizzi R. T., Baum S. A., 1996, MNRAS, 280, 1283

LaMassa S. M., Heckman T. M., Ptak A., Martins L., Wild V., Sonnentrucker P., Hornschemeier A., 2011, ApJ, 729, 52

Lena D., 2014, preprint (arXiv:1409.8264)

Lena D., Robinson A., Seelig T., Schnorr-Müller A., Riffel R. A., StorchiBergmann T., Couto G., 2014, AAS, 223, 25107

Lena D. et al., 2015, ApJ, 806, 84

Lena D., Robinson A., Storchi-Bergmann T., Couto G. S., Schnorr-Müller A., Riffel R. A., 2016, MNRAS, 459, 4485

Li J., Ostriker J., Sunyaev R., 2013, ApJ, 767, 105

Lin M.-Y. et al., 2016, MNRAS, 458, 1375

Lutz D., Maiolino R., Spoon H. W. W., Moorwood A. F. M., 2004, A\&A, 418,465

Malkan M. A., Gorjian V., Tam R., 1998, ApJS, 117, 25

Markwardt C. B., 2009, in Bohlender D. A., Durand D., Dowler P., eds, ASP Conf. Ser. Vol. 411, Astronomical Data Analysis Software and Systems XVIII. Astron. Soc. Pac., San Francisco, p. 251

Mingo B., Hardcastle M. J., Croston J. H., Evans D. A., Hota S., Kharb P., Kraft R. P., 2011, ApJ, 731, 21

Mor R., Netzer H., Elitzur M., 2009, ApJ, 705, 298

Mulchaey J. S., Wilson A. S., Tsvetanov Z., 1996, ApJS, 102, 309

Müller Sánchez F., Davies R. I., Genzel R., Tacconi L. J., Eisenhauer F., Hicks E. K. S., Friedrich S., Sternberg A., 2009, 691, 749

Müller Sánchez F., Prieto M. A., Hicks E. K. S., Vives-Arias H., Davies R. I., Malkan M., Tacconi L. J., Genzel R., 2011, ApJ, 739, 69

Müller Sánchez F., Hicks E. K. S., Malkan M., Davies R., Yu P. C., Shaver S., Davis B., 2017, ApJ, preprint (arXiv:1705.06678)

Mundell C. G., Ferruit P., Nagar N., Wilson A. S., 2009, ApJ, 703, 802

Nagar N. M., Wilson A. S., Mulchaey J. S., Gallimore J. F., 1999, ApJS, 120,209

Nazarova L. S., O’Brien P. T., Ward M. J., 1996, A\&A, 307, 365

Neff S. G., de Bruyn A. G., 1983, A\&A, 128, 318

Nenkova M., Sirocky M. M., Ivezić Ž., Elitzur M., 2008, ApJ, 685, 147

Osterbrock D. E., Ferland G. J., 2006, Astrophysics of Gaseous Nebulae and Active Galactic Nuclei, 2nd edn. University Science Books, Sausalito, CA

Osterbrock D. E., Koski A. T., 1976, MNRAS, 176, 61P 
Peterson B. M. et al., 2004, ApJ, 613, 682

Quillen A. C., Alonso-Herrero A., Rieke M. J., Rieke G. H., Ruiz M., Kulkarni V., 1999, ApJ, 527, 696

Riffel R. A., 2010, Ap\&SS, 327, 239

Riffel R. A., Storchi-Bergmann T., 2011a, MNRAS, 411, 469

Riffel R. A., Storchi-Bergmann T., 2011b, MNRAS, 417, 2752

Riffel R. A., Storchi-Bergmann T., Winge C., Barbosa F. K. B., 2006, MNRAS, 373, 2

Riffel R. A., Storchi-Bergmann T., Winge C., McGregor P. J., Beck T., Schmitt H., 2008, MNRAS, 385, 1129

Riffel R. A., Storchi-Bergmann T., Winge C., 2013, MNRAS, 430, 2249

Riffel R. A., Storchi-Bergmann T., Riffel R., 2014, ApJ, 780, L24

Riffel R. A., Storchi-Bergmann T., Riffel R., 2015, MNRAS, 451, 3587

Riffel R. A., Storchi-Bergmann T., Riffel R., Dahmer-Hahn L. G., Diniz M. R., Schonell A. J., Dametto N. Z., 2017, MNRAS, 470, 992

Schmitt H. R., Kinney A. L., 2000, ApJS, 128, 479

Schmitt H. R., Ulvestad J. S., Antonucci R. R. J., Kinney A. L., 2001, ApJS, 132,199

Schmitt H. R., Donley J. L., Antonucci R. R. J., Hutchings J; B., Kinney A. L., 2003, ApJS, 148, 327

Schnorr-Müller A., Storchi-Bergmann T., Riffel R. A., Ferrari F., Steiner J. E., Axon D. J., Robinson A., 2011, MNRAS, 413, 149

Schnorr-Müller A., Storchi-Bergmann T., Nagar N. M., Ferrari F., 2014a, MNRAS, 438, 3322

Schnorr-Müller A., Storchi-Bergmann T., Nagar N. M., Robinson A., Lena D., Riffel R. A., 2014b, MNRAS, 437, 1708

Simpson C., Mulchaey J. S., Wilson A. S., Ward M. J., Alonso-Herrero A., 1996, ApJ, 457, L19
Smirnova A. A., Moiseev A. V., Afanasiev V. L., 2010, MNRAS, 408, 400

Stoklasová I., Ferruit P., Emsellem E., Jungwiert B., Pécontal E., Sánchez S. F., 2009, A\&A, 500, 1287

Storchi-Bergmann T., Wilson A. S., Baldwin J. A., 1992, ApJ, 396, 45

Storchi-Bergmann T., Lopes R. D. S., McGregor P. J., Riffel R. A., Beck T., Martini P., 2010, MNRAS, 402, 819

Su B. M., Muxlow T. W. B., Pedlar A., Holloway A. J., Steffen W., Kukula M. J., Mutel R. L., 1996, MNRAS, 279, 1111

Tacconi L. J., Genzel R., Blietz M., Cameron M., Harris A. I., Madden S., 1994, ApJ, 426, L77

Tsai M., Hwang C. Y., 2015, AJ, 150, 43

Ueda Y., Ishisaki Y., Takahashi T., Makishima K., Ohashi T., 2001, ApJS, 133,1

Ulvestad J. S., Wilson A. S., 1984, ApJ, 285, 439

Ulvestad J. S., Wrobel J. M., Roy A. L., Wilson A. S., Falcke H., Krichbaum T. P., 1999, ApJ, 517, L81

Whittle M., Pedlar A., Meurs E. J. A., Unger S. W., Axon D. J., Ward M. J., 1988, ApJ, 326, 125

Wilson A. S., Tsvetanov Z. I., 1994, AJ, 107, 1227

Wylezalek D. et al., 2017, MNRAS, 467, 2612

\section{APPENDIX A: EMISSION-LINE FLUX DISTRIBUTIONS}

In this section, we present the flux distributions $\mathrm{H} \beta$, [O I $] \lambda 6300$, $\left[\mathrm{N}\right.$ II] $\lambda 6583$, and $\left[\mathrm{S}_{\mathrm{II}}\right] \lambda 6731$ emission lines for Mrk 6, Mrk 79, Mrk 348, Mrk 607, and Mrk 1058. 

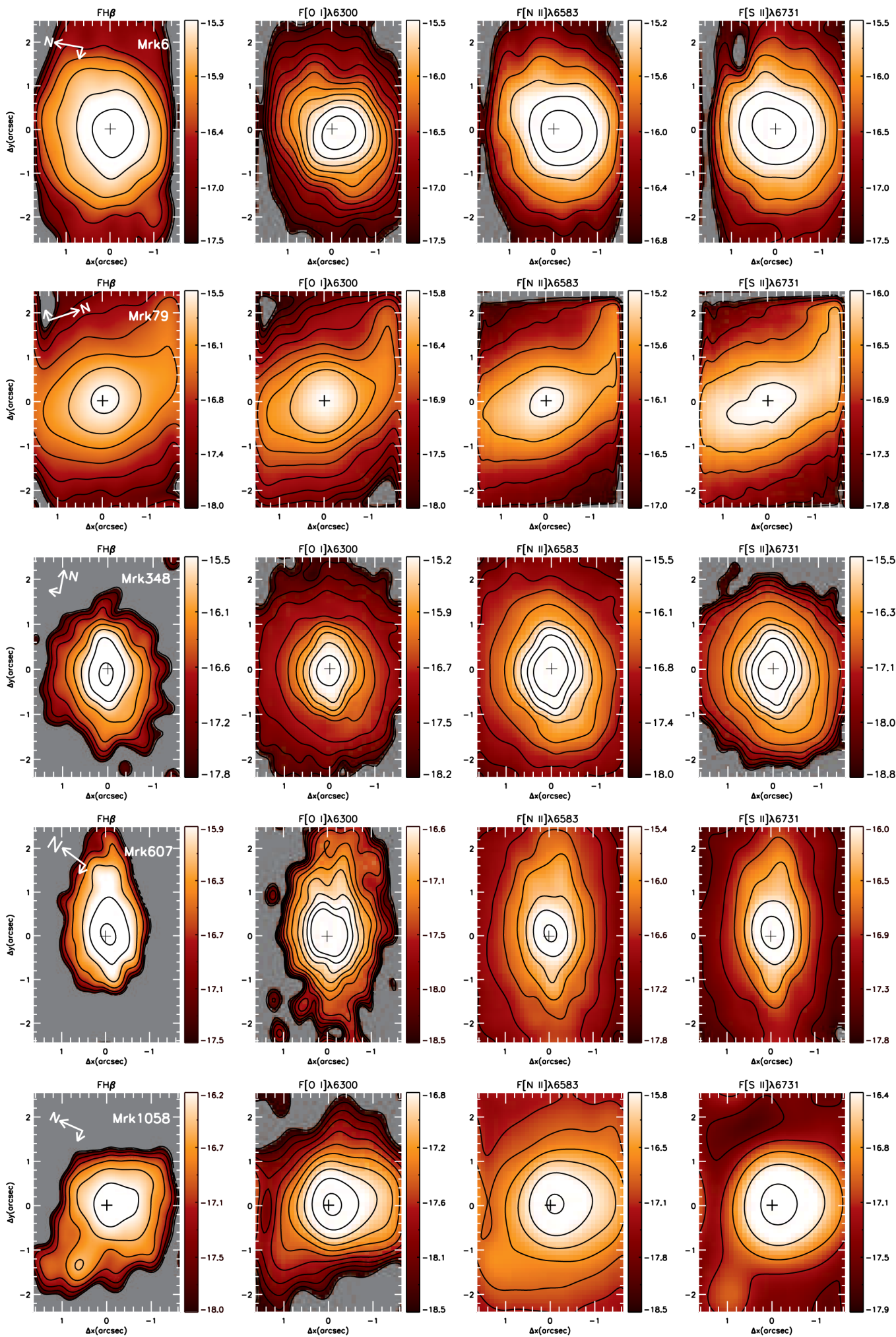

Figure A1. Flux distributions for $\mathrm{H} \beta$, [O I ] $\lambda 6300 \AA$ [N II] $\lambda 6583 \AA$, and [S II] $\lambda 6731 \AA$ A emission lines. The colour bars show the flux scale in logarithmic units of erg s ${ }^{-1} \mathrm{~cm}^{-2}$. From top to bottom: Mrk 6, Mrk 79, Mrk 348, Mrk 607, and Mrk 1058.

This paper has been typeset from a $\mathrm{T}_{\mathrm{E}} \mathrm{X} / \mathrm{L} \mathrm{T} \mathrm{E} \mathrm{X}$ file prepared by the author. 